\title{
ON THE LARGEST KRONECKER AND LITTLEWOOD-RICHARDSON COEFFICIENTS
}

\author{
IGOR PAK ${ }^{\star}$, GRETA PANOVA ${ }^{\dagger}$ AND DAMIR YELIUSSIZOV*
}

\begin{abstract}
We give new bounds and asymptotic estimates for Kronecker and LittlewoodRichardson coefficients. Notably, we resolve Stanley's questions on the shape of partitions attaining the largest Kronecker and Littlewood-Richardson coefficients. We apply the results to asymptotics of the number of standard Young tableaux of skew shapes.
\end{abstract}

\section{INTRODUCTION}

What is largest dimension $\mathbf{D}(n)$ of an irreducible representation of $S_{n}$ ? Which partitions correspond to the largest representations? These questions are both classical and surprisingly challenging. They have been heavily studied in both combinatorics and probability, in connection to the longest increasing subsequences. We refer to [AD, BDJ, Rom for the history of this problem.

In algebraic combinatorics and related fields, the Kronecker and Littlewood-Richardson (LR-) coefficients play a crucial role. They are the structure constants in the ring of characters of $S_{n}$ and $\mathrm{GL}_{N}(\mathbb{C})$, respectively:

$$
\chi^{\mu} \cdot \chi^{\nu}=\sum_{\nu} g(\lambda, \mu, \nu) \chi^{\lambda} \quad \text { and } \quad s_{\mu} \cdot s_{\nu}=\sum_{\lambda} c_{\mu, \nu}^{\lambda} s_{\lambda} .
$$

These coefficients have been intensely studied from combinatorial (see e.g. [S4, vL]), geometric (see e.g. [Ful]), probabilistic (see [Bia]), and computational point of view (see e.g. [Bür, BI]). Yet, relatively little is known about the asymptotics of these coefficients (see \$7.1).

In recent years, Stanley computed asymptotic of the largest Kronecker and LR-coefficients:

Theorem 1.1 (Stanley [S5, S6]). We have:

$$
\text { (**) } \quad \max _{0 \leq k \leq n} \max _{\lambda \vdash n} \max _{\mu \vdash k} \max _{\nu \vdash n-k} c_{\mu, \nu}^{\lambda}=2^{n / 2-O(\sqrt{n})} .
$$

Having obtained these interesting asymptotics, Stanley asked in [S5, [S6]: What partitions attain these maxima? His original proof used certain summation identities which gave no indication on how to answer either question 1

In this paper we largely resolve questions for both $(*)$ and $(* *)$, and the answers turn out to be the Vershik-Kerov-Logan-Shepp (VKLS) shapes. These are the shapes that attain the largest dimension $\mathbf{D}(n)$ mentioned above. The precise formulations of both results is

\footnotetext{
April 26, 2018.

${ }^{\star}$ Department of Mathematics, UCLA, Los Angeles, CA 90095. Email: \{pak, damir\}@math.ucla.edu.

${ }^{\dagger}$ Department of Mathematics, UPenn, Philadelphia, PA 19104, and IAS, Princeton, NJ 08540.

Email: panova@math.upenn.edu.

${ }^{1}$ R. P. Stanley, personal communication (2017).
} 
somewhat technical (see next section), with notable differences between two cases. Contrary to the many other questions in the area, the Kronecker coefficients are actually easier to understand than the LR-coefficients, for reasons that will become apparent only much later in the paper (see \$7.2).

Somewhat surprisingly, and in contrast with the technical proofs in the earlier results, we are able to resolve both questions by using basic character estimates and utilizing various existing technical results in the area.

Throughout the paper, we worked hard to simplify and streamline the proofs, and remove $S_{n}$-specific tools to make sure that our bounds can be extended to general finite groups. In the companion paper [PPY], we present slightly weaker but much more general bounds for induced multiplicities, which extend both the Kronecker and the LR-coefficients (cf. \$7.2).

1.1. Kronecker coefficients. Recall that the largest dimension $\mathbf{D}(n):=\max _{\lambda \vdash n} f^{\lambda}$ satisfies

$$
\sqrt{n !} e^{-c_{1} \sqrt{n}(1+o(1))} \leq \mathbf{D}(n) \leq \sqrt{n !} e^{-c_{2} \sqrt{n}(1+o(1))}
$$

for some $c_{1}>c_{2}>0$ [VK2]. This motivates the following definition.

Definition 1.2. A sequence of partitions $\left\{\lambda^{(n)} \vdash n\right\}$ is called Plancherel if for some $a>0$, we have:

$$
f^{\lambda^{(n)}} \geq \sqrt{n !} e^{-a \sqrt{n}} \text { for all } n \text {. }
$$

Theorem 1.3 ([VK1] and [LoS]). Every Plancherel partition sequence has VKLS shape.

See $₫ 2.5$ for precise definitions of VKLS shapes. We write $f^{\lambda^{(n)}}=\sqrt{n !} e^{-O(\sqrt{n})}$ when we do not want to specify the constant implied by the $O(\cdot)$ notation.

Theorem 1.4. Let $\left\{\lambda^{(n)} \vdash n\right\},\left\{\mu^{(n)} \vdash n\right\},\left\{\nu^{(n)} \vdash n\right\}$ be three partitions sequences, such that

$$
g\left(\lambda^{(n)}, \mu^{(n)}, \nu^{(n)}\right)=\sqrt{n !} e^{-O(\sqrt{n})} .
$$

Then all three partition sequences are Plancherel. Conversely, for every two Plancherel partition sequences $\left\{\lambda^{(n)} \vdash n\right\}$ and $\left\{\mu^{(n)} \vdash n\right\}$, there exists a Plancherel partition sequence $\left\{\nu^{(n)} \vdash n\right\}$, s.t. (1.1) holds.

This resolves Stanley's question on Kronecker coefficients as it implies that $\mathbf{K}(n)$ must be attained at VKLS shapes. We also obtain the several extensions and refinements of these results.

\subsection{Littlewood-Richardson coefficients. Define}

$$
\mathbf{C}(n, k):=\max _{\lambda \vdash n} \max _{\mu \vdash k} \max _{\nu \vdash n-k} c_{\mu, \nu}^{\lambda} .
$$

Theorem 1.5. For all $n>k \geq 1$, we have:

$$
\sqrt{\left(\begin{array}{l}
n \\
k
\end{array}\right)} e^{-d \sqrt{n}} \leq \mathbf{C}(n, k) \leq \sqrt{\left(\begin{array}{l}
n \\
k
\end{array}\right)}
$$

for some universal constant $d>0$.

This refines Stanley's asymptotic bound $(* *)$ in Theorem 1.1. Note that $\left(\begin{array}{l}n \\ k\end{array}\right)$ is the dominant term of the asymptotics for all $\sqrt{n} \leq k \leq n-\sqrt{n}$. Note also that $\{\mathbf{C}(n, k)\}$ are not unimodal

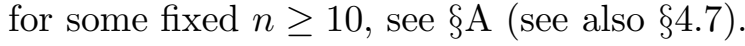


Theorem 1.6. Fix $0<\theta<1$ and let $k_{n}:=\lfloor\theta n\rfloor$. Then:

(i) for every Plancherel partition sequence $\left\{\lambda^{(n)} \vdash n\right\}$, there exist Plancherel partition sequences $\left\{\mu^{(n)} \vdash k_{n}\right\}$ and $\left\{\nu^{(n)} \vdash n-k_{n}\right\}$, s.t.

$$
(*) \quad c_{\mu^{(n)}, \nu^{(n)}}^{\lambda^{(n)}}=\left(\begin{array}{c}
n \\
k_{n}
\end{array}\right)^{1 / 2} e^{-O(\sqrt{n})},
$$

(ii) for all Plancherel partition sequences $\left\{\mu^{(n)} \vdash k_{n}\right\}$ and $\left\{\nu^{(n)} \vdash n-k_{n}\right\}$, there exists a Plancherel partition sequence $\left\{\lambda^{(n)} \vdash n\right\}$, s.t. $(*)$ holds,

(iii) for all Plancherel partition sequences $\left\{\lambda^{(n)} \vdash n\right\}$ and $\left\{\mu^{(n)} \vdash k_{n}\right\}$, there exists a partition sequence $\left\{\nu^{(n)} \vdash n-k_{n}\right\}$, s.t.

$$
f^{\nu^{(n)}}=\sqrt{n !} e^{-O\left(n^{2 / 3} \log n\right)} \quad \text { and } \quad c_{\mu^{(n)}, \nu^{(n)}}^{\lambda^{(n)}}=\left(\begin{array}{c}
n \\
k_{n}
\end{array}\right)^{1 / 2} e^{-O\left(n^{2 / 3} \log n\right)} .
$$

Combined, these results resolve Stanley's question on LR-coefficients. Note that Proposition 4.16 shows that one cannot conclude that LR-coefficients are large when the dimensions of all three representations are large. Note also that part (iii) requires the bound to hold in a wider setting than $(i)$. It is thus unsurprising that it has weaker conclusions.

We also obtain a partial converse:

Theorem 1.7. Fix $\theta=1 / 2$ and let $k_{n}:=\lfloor\theta n\rfloor$. Suppose $\left\{\lambda^{(n)} \vdash n\right\},\left\{\mu^{(n)} \vdash k_{n}\right\}$ and $\left\{\nu^{(n)} \vdash n-k_{n}\right\}$ be three partitions sequences, s.t.

$$
c_{\mu^{(n)}, \nu^{(n)}}^{\lambda^{(n)}}=\left(\begin{array}{c}
n \\
n / 2
\end{array}\right)^{1 / 2} e^{-O(n / \log n)}
$$

Then all three partition sequences satisfy:

$$
f^{\lambda^{(n)}}=\sqrt{n !} e^{-O(n)}, \quad f^{\mu^{(n)}}=\sqrt{(n / 2) !} e^{-O(n)} \quad \text { and } \quad f^{\nu^{(n)}}=\sqrt{(n / 2) !} e^{-O(n)} .
$$

1.3. Structure of the paper. We start with definitions and known results in Section 2 . Kronecker coefficients and the proof of Theorem 1.4 are given in Section 3. We continue with a lengthy Section 4 on LR-coefficients, including the proof of Theorem 1.5 and parts $(i)$, (ii) of Theorem 1.6. We continue with a short but technical Section 5, again on LR-coefficients, where we prove Theorem 1.7. We apply our results in Section 6 to the estimate the number of standard Young tableaux of skew shape, proving the remaining part (ii) of Theorem 1.6. We conclude with final remarks and open problems (Section 7).

\section{Definitions, notation and BaCkground Results}

2.1. Partitions. Let $p(n)=\#\{\lambda \vdash n\}$ be the number of partitions of $n$. The HardyRamanujan asymptotic formula gives:

$$
p(n) \sim \frac{1}{4 n \sqrt{3}} e^{\pi \sqrt{\frac{2 n}{3}}} \text { as } n \rightarrow \infty .
$$

We also need the log-concavity of the partition function:

$$
p(n-k) \cdot p(n+k) \geq p(n)^{2},
$$

for all $n>25, k=1$, and for all $n>k>1[\mathrm{DP}]$. 
2.2. Dimensions and Young tableaux. We assume the reader is familiar with standard definition and notation in the area; we follow [S4] in most cases.

Let $f^{\lambda / \mu}=\# \operatorname{SYT}(\lambda / \mu)$ denote the number of standard Young tableaux of skew shape $\lambda / \mu$. Recall the hook-length formula (see e.g. [S4, §7.21]):

$$
f^{\lambda}=n ! \prod_{(i, j) \in \lambda} \frac{1}{h_{i j}},
$$

where $n=|\lambda|$ and $h_{i j}=\lambda_{i}+\lambda_{j}^{\prime}-i-j+1$ is the hook length. For skew shapes, we have the following corollary of the Naruse hook-length formula:

$$
f^{\lambda / \mu} \geq n ! \prod_{(i, j) \in \lambda / \mu} \frac{1}{h_{i j}},
$$

where $n=|\lambda / \mu|$, see [MPP1].

Let $s_{\lambda}\left(x_{1}, x_{2}, \ldots\right)$ denote the Schur function. For the evaluations $s_{\lambda}\left(1^{m}\right):=s_{\lambda}(1, \ldots, 1)$, $m$ ones, we have the following hook-content formula:

$$
s_{\lambda}\left(1^{m}\right)=\prod_{(i, j) \in \lambda} \frac{m+j-i}{h_{i j}} .
$$

(see e.g. [S4, $\S 7.21]$ ).

2.3. Largest dimension. Vershik and Kerov [VK2 proved that for all $n$ large enough:

$$
\sqrt{n !} e^{-c_{1} \sqrt{n}(1+o(1))} \leq \mathbf{D}(n) \leq \sqrt{n !} e^{-c_{2} \sqrt{n}(1+o(1))},
$$

where

$$
c_{1}=\pi \sqrt{\frac{1}{6}} \approx 1.2825 \text { and } c_{2}=\frac{\pi-2}{\pi^{2}} \approx 0.1157 .
$$

See [OEIS, A003040] and $9 \mathrm{~A}$ for the initial values and [VP] for numerical experiments. Note that the lower bound follows from the Burnside identity:

$$
\sum_{\lambda \vdash n}\left(f^{\lambda}\right)^{2}=n !
$$

However, the upper bound is rather remarkable. In fact, Vershik and Kerov proved in VK2] that with respect to the Plancherel measure $\left(f^{\lambda}\right)^{2} / n$ !, almost all dimensions $f^{\lambda}$ lie in the interval in (2.4). Moreover, in the same setting, Bufetov proved [Buf]:

$$
f^{\lambda}=\sqrt{n !} e^{-h \sqrt{n}(1+o(1))} \quad \text { a.s., for some } c_{1} \geq h \geq c_{2} .
$$

This should be compared with the Vershik-Kerov-Pass conjecture:

$$
\mathbf{D}(n)=\sqrt{n !} e^{-a \sqrt{n}(1+o(1))}
$$

for some $c_{2} \leq a \leq c_{1}$. This was implicitly suggested by Vershik in Ver] (in greater generality), and formally stated by Kerov and Pass [KP]. 
2.4. Stable limit shapes. The following definition follows MPP1]. Let $\omega:[0, a] \rightarrow[0, b]$ be a non-increasing continuous function. Suppose sequence of partitions $\left\{\lambda^{(n)}\right\}$ satisfies the following property

$$
(\sqrt{n}-L) \omega<\left[\lambda^{(n)}\right]<(\sqrt{n}+L) \omega, \quad \text { for some } L>0,
$$

where we write $[\lambda]$ to denote a function giving the boundary of Young diagram $\lambda$. In this case we say that a sequence of partitions $\left\{\lambda^{(n)}\right\}$ has a strongly stable shape $\omega$, and write $\lambda^{(n)} \mapsto \omega$.

Suppose we are given two stable shapes $\omega, \pi:[0, a] \rightarrow[0, b]$, such that $\pi(x) \leq \omega(x)$ for all $x \geq 0$. To simplify the notation, denote by $\mathcal{C}=\mathcal{C}(\omega / \pi) \subset \mathbb{R}_{+}^{2}$ the region between the curves. One can view $\mathcal{C}$ as the stable shape of skew shapes, and denote by area $(\omega / \pi)$ the area of $\mathcal{C}$.

Define the hook function $\hbar: \mathcal{C} \rightarrow \mathbb{R}_{+}$to be the scaled function of the hooks: $\hbar(x, y):=$ $h(\lfloor x \sqrt{n}\rfloor,\lfloor y \sqrt{n}\rfloor)$.

Theorem 2.1 ([MPT] and [MPP1, §6]). In notation above, suppose $\lambda^{(n)} \mapsto \omega, \mu^{(n)} \mapsto \pi$ and area $(\omega / \pi)=1$. Then we have:

$$
f^{\lambda^{(n)} / \mu^{(n)}}=\sqrt{n !} e^{c(\omega / \pi) n+o(n)},
$$

for some constant $c(\omega / \pi)$ satisfying

$$
\Upsilon(\omega / \pi)-\frac{1}{2} \leq c(\omega / \pi) \leq \Upsilon(\omega / \pi)-\frac{1}{2}+\log 2,
$$

where

$$
\Upsilon(\omega / \pi):=-\iint_{\mathcal{C}} \log \hbar(x, y) d x d y
$$

The constant $\Upsilon(\omega / \pi)$ in the theorem is called the hook integral of the limit shape. The lower bound on $c(\omega / \pi)$ in the theorem follows easily from (2.2), but the upper bound requires full power of the Naruse hook-length formula [MPP1].

2.5. VKLS shapes. A partition sequence $\left\{\lambda^{(n)}\right\}$ is said to have Vershik-Kerov-Logan-Shepp (VKLS) shape if

$$
\left|\frac{1}{\sqrt{n}}\left[\lambda^{(n)}\right]-\psi\right|<C n^{1 / 6} \text { for some } C>0,
$$

where $\psi:[0,2] \rightarrow[0,2]$ is obtained by $135^{\circ}$ rotation of the curve $(x, \varphi(x))$, defined by

$$
\varphi(x):=\frac{2}{\pi}\left(x \arcsin \frac{x}{\sqrt{2}}+\sqrt{2-x^{2}}\right), \quad-\sqrt{2} \leq x \leq \sqrt{2} .
$$

Here the distance $|\cdot|$ is the Fréchet distance between the curves.

It follows from Theorem 1.3, that $\psi$ is the unique curve of area 1, s.t. $c(\psi)=0$ and $\Upsilon(\psi)=1 / 2$. The latter holds since the lower bound in (2.2) is an equality for straight shapes by the hook-length formula (2.1). This implies:

Proposition 2.2. Suppose a partition sequence $\left\{\lambda^{(n)}\right\}$ has a strongly stable shape $\omega$, and satisfies

Then $\omega=\varphi$ defined in (2.7).

$$
f^{\lambda^{(n)}}=\sqrt{n !} e^{-o(n)}
$$

We refer to [Rom for the detailed proof of the VKLS theorem (Theorem 1.3), and its many interesting applications. 


\section{KRONECKER COEFFICIENTS}

3.1. General inequalities. The Kronecker coefficient $g(\lambda, \mu, \nu)$ defined in the introduction satisfy:

$$
g(\lambda, \mu, \nu)=\left\langle\chi^{\lambda}, \chi^{\mu} \chi^{\nu}\right\rangle=\left\langle\chi^{\lambda} \chi^{\mu} \chi^{\nu}, 1\right\rangle .
$$

This implies the symmetries

$$
g(\lambda, \mu, \nu)=g(\lambda, \nu, \mu)=g(\mu, \lambda, \nu)=\ldots
$$

In particular, we have a general upper bound:

$$
g(\lambda, \mu, \nu) \leq f^{\lambda} \cdot \min \left\{f^{\mu} / f^{\nu}, f^{\nu} / f^{\mu}\right\} \leq f^{\lambda} .
$$

Proposition 3.1. Let $\lambda, \mu, \nu \vdash n$. Suppose $g(\lambda, \mu, \nu) \geq \mathbf{D}(n) / a$, for some $a \geq 1$. Then: $f^{\lambda}, f^{\mu}, f^{\nu} \geq \mathbf{D}(n) / a$.

Proof. This follows immediately from (3.2) and the symmetries (3.1).

3.2. Largest Kronecker coefficients. Define the largest Kronecker coefficient of $G$ as follows:

Similarly, define

$$
\mathbf{K}(n):=\max _{\lambda, \mu, \nu \vdash n} g(\lambda, \mu, \nu) .
$$

$$
\mathbf{A}(n):=\sum_{\lambda, \mu, \nu \vdash n} g(\lambda, \mu, \nu)^{2}
$$

Lemma 3.2. We have:

$$
\mathbf{A}(n)=\sum_{\alpha \vdash n} z_{\alpha}
$$

where $z_{\alpha}=|C(\alpha)|$ is the size of the centralizer of an element $x \in \alpha$.

Proof of Lemma 3.2. We have:

$$
g(\lambda, \mu, \nu)=\frac{1}{n !} \sum_{x \in G} \chi^{\lambda}(w) \chi^{\mu}(x) \chi^{\nu}(x) .
$$

Hence, we can write the sum of squares as

$$
\begin{aligned}
& \sum_{\lambda, \mu, \nu \vdash n} g(\lambda, \mu, \nu)^{2}=\frac{1}{n !^{2}} \sum_{x, y \in S_{n}} \sum_{\lambda \vdash n} \chi^{\lambda}(x) \chi^{\lambda}(y) \sum_{\mu \vdash n} \chi^{\mu}(x) \chi^{\mu}(y) \sum_{\nu \vdash n} \chi^{\nu}(x) \chi^{\nu}(y) \\
& =\frac{1}{n !^{2}} \sum_{x, y \in S_{n}}\left(\sum_{\lambda \vdash n} \chi^{\lambda}(x) \chi^{\lambda}(y)\right)^{3}=\frac{1}{n !^{2}} \sum_{\alpha \vdash n}\left(\frac{n !}{z_{\alpha}}\right)^{2}\left(z_{\alpha}\right)^{3}=\sum_{\alpha \vdash n} z_{\alpha} .
\end{aligned}
$$

Here the last equality follows from the orthogonality of characters.

Proposition 3.3. We have:

$$
\frac{\sqrt{n !}}{p(n)^{3 / 2}} \leq \mathbf{K}(n) \leq \mathbf{D}(n) .
$$

Proof. In (3.3), we have RHS $\geq z_{1}=n$ !. This gives the lower bound. The upper bound follows from (3.2). 
The following result can be viewed as a converse of Proposition 3.1 .

Theorem 3.4. Let $\mu, \nu \vdash n$. Suppose $f^{\mu}, f^{\nu} \geq \mathbf{D}(n) / a$ for some $a \geq 1$. Then there exist $\lambda \vdash n$, s.t.

$$
f^{\lambda} \geq \frac{\mathbf{D}(n)}{a \sqrt{p(n)}} \quad \text { and } \quad g(\lambda, \mu, \nu) \geq \frac{\mathbf{D}(n)}{a^{2} p(n)} .
$$

Proof. Let $\lambda \vdash n$ be the partition of the largest term in the RHS of

$$
\frac{\mathbf{D}(n)^{2}}{a^{2}} \leq f^{\mu} \cdot f^{\nu}=\sum_{\lambda \vdash n} g(\lambda, \mu, \nu) f^{\lambda} .
$$

On the one hand,

On the other hand,

$$
g(\lambda, \mu, \nu) \geq \frac{1}{p(n) \cdot \mathbf{D}(n)} \cdot \frac{\mathbf{D}(n)^{2}}{a^{2}}=\frac{\mathbf{D}(n)}{a^{2} p(n)} .
$$

which implies the result.

$$
\left(f^{\lambda}\right)^{2} \geq g(\lambda, \mu, \nu) f^{\lambda} \geq \frac{1}{p(n)} \cdot \frac{\mathbf{D}(n)^{2}}{a^{2}}
$$

3.3. Refined Kronecker coefficients. Fix $\lambda, \mu \vdash n$. Define the largest refined Kronecker coefficient

Clearly, $\mathbf{K}(\lambda, \mu) \leq \mathbf{K}(n)$.

$$
\mathbf{K}(\lambda, \mu):=\max _{\nu \vdash n} g(\lambda, \mu, \nu)
$$

Proposition 3.5. For all $\lambda, \mu \vdash n$, we have:

$$
\frac{f^{\lambda} f^{\mu}}{\sqrt{p(n) n !}} \leq \mathbf{K}(\lambda, \mu) \leq \min \left\{f^{\lambda}, f^{\mu}\right\}
$$

Proof. Let

$$
A(\lambda, \mu):=\sum_{\nu \vdash n} g(\lambda, \mu, \nu)^{2} .
$$

Recall Burnside's identity (2.6) and

$$
\sum_{\nu \vdash n} g(\lambda, \mu, \nu) f^{\nu}=f^{\mu} f^{\lambda}
$$

Now apply the Cauchy-Schwarz inequality to vectors $\left(f^{\nu}\right),(g(\lambda, \mu, \nu)) \in \mathbb{R}^{p(n)}$, both indexed by $\nu \vdash n$. We obtain:

$$
A(\lambda, \mu) \geq \frac{\left(f^{\lambda}\right)^{2}\left(f^{\mu}\right)^{2}}{n !} .
$$

Therefore, for the maximal term in the summation $A(\lambda, \mu)$, we have:

$$
\max _{\nu \vdash n} g(\lambda, \mu, \nu) \geq \frac{f^{\lambda} f^{\mu}}{\sqrt{p(n) n !}} .
$$

This gives the lower bound. The upper bound follows from (3.2).

Corollary 3.6. For all $n \in \mathbb{N}$, have:

$$
\frac{\mathbf{D}(n)^{2}}{\sqrt{p(n) n !}} \leq \mathbf{K}(n) \leq \mathbf{D}(n) .
$$


Proof. In Proposition 3.5, take $\lambda, \mu \vdash n$ such that $f^{\lambda}=f^{\mu}=\mathbf{D}(n)$. This gives the lower bound. The upper bound is given in Proposition 3.3 .

Note that the lower bound in Corollary 3.6 is slightly stronger than the lower bound in Proposition 3.3. Now the bounds (2.4) give us:

Corollary 3.7. We have:

$$
\sqrt{n !} e^{-3 c_{1} \sqrt{n}(1+o(1))} \leq \mathbf{K}(n) \leq \sqrt{n !} e^{-c_{2} \sqrt{n}(1+o(1))},
$$

where $c_{1}, c_{2}>0$ are given in (2.5).

It would be interesting to improve either of these two bounds.

Remark 3.8. The following asymptotic formula is well known and easy to see OEIS, A110143:

$$
\mathbf{A}(n)=\sum_{\alpha \vdash n} z_{\alpha}=n !\left(1+\frac{2}{n^{2}}+O\left(\frac{1}{n^{3}}\right)\right) .
$$

This shows that the lower bounds (3.4) in the proof of Proposition 3.5 are also asymptotically sharp. Indeed, in the notation of the proof of the proposition, we have:

$$
\mathbf{A}(n)=\sum_{\lambda, \mu, \nu \vdash n} g(\lambda, \mu, \nu)^{2}=\sum_{\lambda, \mu \vdash n} A(\lambda, \mu) \geq \sum_{\lambda, \mu \vdash n} \frac{\left(f^{\lambda}\right)^{2}\left(f^{\mu}\right)^{2}}{n !}=n !,
$$

which implies that the inequalities (3.4) are sharp up to a small (additive) error.

\subsection{Shape of the largest Kronecker coefficients.}

Proof of Theorem 1.4. The theorem is a special case of Theorem 3.4, where $a=e^{-O(\sqrt{n})}$. Indeed, both parts of Theorem 1.4 either assume or require

$$
g\left(\lambda^{(n)}, \mu^{(n)}, \nu^{(n)}\right), f^{\lambda^{(n)}}, f^{\mu^{(n)}}, f^{\nu^{(n)}} \geq \sqrt{n !} e^{-\alpha \sqrt{n}}
$$

for some $\alpha \geq 0$, implied by the $O(\cdot)$ notation in the theorem. Theorem 3.4 makes this dependence precise.

Note that the proof of Theorem 3.4 is simple enough to imply a quantitative version of Biane's concentration of characters result [Bia].

Proposition 3.9. Let $\alpha, \beta, \varepsilon>0$ such that $\alpha+\beta>c_{1}$. Let $\lambda, \mu \vdash n$ such that

$$
f^{\lambda}, f^{\mu}>\sqrt{n !} e^{-\alpha \sqrt{n}} \text {. }
$$

Then:

$$
\sum_{\nu \in \mathcal{P}_{\beta}(n)} g(\lambda, \mu, \nu) f^{\nu}>(1-\varepsilon) f^{\lambda} f^{\mu} \quad \text { for all } n \text { large enough, }
$$

where the summation is over the set $\mathcal{P}_{\beta}(n)$ of $\nu$ such that

$$
f^{\nu}>\sqrt{n !} e^{-\beta \sqrt{n}} \text {. }
$$

The proposition is phrased in the form to underscore the similarity with Thm 1.4.1 in [Bia]. Of course, Biane's result is more general as it applied to partitions with other limits shapes $\lambda$, which all must have $f^{\lambda}=\sqrt{n !} e^{O(n)}$, see [MPP1]. 
Proof of Proposition 3.9. We have:

$$
\sum_{\nu \vdash n} g(\lambda, \mu, \nu) f^{\nu}=f^{\lambda} \cdot f^{\mu}>n ! e^{-2 \alpha \sqrt{n}}
$$

while

$$
\sum_{\nu \notin \mathcal{P}_{\beta}(n)} g(\lambda, \mu, \nu) f^{\nu} \leq\left(\sqrt{n !} e^{-\beta \sqrt{n}}\right)^{2} p(n)=n ! e^{-2\left(\beta+c_{1}\right) \sqrt{n}(1+o(1))} .
$$

This implies the result.

3.5. Tensor square conjectures. It was suggested in [HSTZ that for all $n$ large enough, there exists $\lambda \vdash n$, such that $g(\lambda, \lambda, \nu)>0$ for all $\nu \vdash n$. In other words, the tensor square $\chi^{\lambda} \times \chi^{\lambda}$ contains all characters $\chi^{n}$. One would want to start with the Plancherel shape $\lambda$, for which by by Proposition 3.9 the tensor square has large average isotypic components. Note however, that the Plancherel shape is a large class of partitions, making them hard to study. For example, one needs $\lambda=\lambda^{\prime}$ since otherwise $g\left(\lambda, \lambda, 1^{n}\right)=0$, and there are other similar small constraints $\lambda$ must satisfy.

Saxl conjectured [PPV] that $\lambda=(k-1, k-2, \ldots, 1) \vdash n=\left(\begin{array}{c}k \\ 2\end{array}\right)$ for large enough $k$ would have $g(\lambda, \lambda, \nu)>0$ for all $\nu \vdash n$. This is now known for most $\nu \vdash n[\mathrm{LuS}$, but there are only very weak lower bounds on $g(\lambda, \lambda, \nu)$. Even when $\lambda=\nu$, the Bessenrodt-Behns lower bound $g(\lambda, \lambda, \lambda) \geq 1$ is the best bound we have in this case [BB]. In fact, the largest known values of Kronecker coefficients for explicitly given families of partitions are subexponential [PP2].

This all makes the following conjecture both plausible and out of reach. Such a result would provide an ultimate extension in the converse part of Theorem 1.4.

Conjecture 3.10. Let $\left\{\lambda^{(n)} \vdash n\right\},\left\{\mu^{(n)} \vdash n\right\},\left\{\nu^{(n)} \vdash n\right\}$ be three Plancherel partitions sequences. Then:

$$
g\left(\lambda^{(n)}, \mu^{(n)}, \nu^{(n)}\right)=\sqrt{n !} e^{-O(n)} .
$$

Below we show that it is possible to have three large characters with a zero Kronecker coefficient. This shows that assumptions in the conjecture cannot be significantly weakened.

3.6. Vanishing Kronecker coefficients. Let us present examples of partition families of large dimensions with zero Kronecker coefficients

Theorem 3.11 (Regev $[\operatorname{Reg}])$. Let $\lambda, \mu, \nu \vdash n$ such that $\ell(\lambda)>\mu_{1} \cdot \nu_{1}$. Then $g(\lambda, \mu, \nu)=0$.

Corollary 3.12. There is a partition family $\left\{\lambda^{(n)}\right\}$, s.t.

$$
g\left(\lambda^{(n)}, \lambda^{(n)}, \lambda^{(n)}\right)=0 \text { and } f^{\lambda}=\sqrt[3]{n !} e^{O(n)} .
$$

Proof. Let $\lambda=\left(a^{2}\right)^{a-1} \vdash n$, where $n=(a-1) a^{2}$. Note that $\ell(\lambda)>\left(\lambda_{1}\right)^{2}$. Then $g(\lambda, \lambda, \lambda)=0$ by Regev's theorem. The degree follows from the hook-length formula (2.1). We omit the details.

Theorem 3.13 (Dvir [Dvir]). Let $\lambda, \mu, \nu \vdash n$ such that $\ell(\lambda)>|\mu \cap \nu|$. Then $g(\lambda, \mu, \nu)=0$.

Corollary 3.14. There are three partition families $\left\{\lambda^{(n)}\right\},\left\{\mu^{(n)}\right\}$ and $\left\{\nu^{(n)}\right\}$, s.t.

$$
\begin{aligned}
g\left(\lambda^{(n)}, \mu^{(n)}, \nu^{(n)}\right)=0, \quad f^{\lambda^{(n)}} & =(n !)^{\Theta(1)}, \\
f^{\mu^{(n)}}=\sqrt{n !} e^{-O(\sqrt{n})} \quad \text { and } \quad f^{\nu^{(n)}} & =\sqrt{n !} e^{-O(n)} .
\end{aligned}
$$


Proof. Let $\mu \vdash n$ be a Plancherel shape, and $\nu=\left(a^{a}\right)$, where $n=a^{2}$. Let $\lambda=\left(b^{b} 1^{n-b^{2}}\right)$ for $b=\lfloor\varepsilon a\rfloor$, and $\varepsilon>0$ a fixed constant sufficiently small so that $|\mu \cap \nu|<\left(1-\varepsilon^{2}\right) a^{2}$. For example, $\varepsilon=0.2$ works for $n$ large enough. Since

$$
\ell(\lambda)>n-b^{2} \geq\left(1-\varepsilon^{2}\right) a^{2}>|\mu \cap \nu|,
$$

by Dvir's theorem, we have $g(\lambda, \mu, \nu)=0$. The bound on dimension of $f^{\lambda}$ follows by the hook-length formula (2.1) and direct calculation.

\section{LitTleWood-Richardson COEFFicients of $S_{n}$}

4.1. General inequalities. The Littlewood-Richardson coefficients $c_{\mu, \nu}^{\lambda}$ satisfy:

$$
\sum_{\lambda \vdash n} c_{\mu, \nu}^{\lambda} f^{\lambda}=\left(\begin{array}{l}
n \\
k
\end{array}\right) f^{\mu} f^{\nu} \quad \text { and } \quad \sum_{\mu \vdash k, \nu \vdash n-k} c_{\mu, \nu}^{\lambda} f^{\mu} f^{\nu}=f^{\lambda} .
$$

Lemma 4.1. For every $0 \leq k \leq n$, we have:

$$
\sum_{\lambda \vdash n} \sum_{\mu \vdash k, \nu \vdash n-k}\left(c_{\mu, \nu}^{\lambda}\right)^{2} \geq\left(\begin{array}{l}
n \\
k
\end{array}\right) .
$$

Proof. To simplify the notation, let $G=S_{n}$ and $H=S_{k} \times S_{n-k}$. Denote by $\pi^{\mu \nu}=\chi^{\mu} \otimes \chi \nu$, and let $\xi^{\lambda}=\left.\chi^{\lambda}\right|_{H}$ be the restriction of the character $\chi^{\lambda}$ to $H$. We have:

$$
c_{\mu, \nu}^{\lambda}=\sum_{\alpha \in \operatorname{Conj}(H)} z_{\alpha}^{-1} \xi^{\lambda}(\alpha) \pi^{\mu \nu}(\alpha)
$$

Then:

$$
\begin{aligned}
\sum_{\lambda \vdash n} \sum_{\mu \vdash k, \nu \vdash n-k}\left(c_{\mu, \nu}^{\lambda}\right)^{2} & =\sum_{\alpha, \gamma \in \operatorname{Conj}(H)} z_{\alpha}^{-1} z_{\gamma}^{-1} \sum_{\lambda \vdash n} \sum_{\mu \vdash k, \nu \vdash n-k} \xi^{\lambda}(\alpha) \xi^{\lambda}(\gamma) \pi^{\mu \nu}(\alpha) \pi^{\mu \nu}(\gamma) \\
& =\sum_{\alpha \in \operatorname{Conj}(H)} z_{\alpha}(H)^{-2}\left(z_{\alpha}(H) \cdot z_{\alpha}(G)\right)=\sum_{\alpha \in \operatorname{Conj}(H)} \frac{z_{\alpha}(G)}{z_{\alpha}(H)},
\end{aligned}
$$

where $z_{\alpha}(H)=\left|C_{H}(x)\right|$ denotes the size of the centralizer of $x \in \alpha$ within $H$, and $z_{\alpha}(G)=$ $\left|C_{G}(x)\right|$ is the size of the centralizer within $G$. Since the RHS $\geq z_{1}(G) / z_{1}(H)=\left(\begin{array}{l}n \\ k\end{array}\right)$, we obtain the inequality.

Lemma 4.2. For every $0 \leq k \leq n$, we have:

$$
\sum_{\lambda \vdash n}\left(c_{\mu, \nu}^{\lambda}\right)^{2} \leq\left(\begin{array}{l}
n \\
k
\end{array}\right) \quad \text { and } \quad \sum_{\mu \vdash k} \sum_{\nu \vdash n-k}\left(c_{\mu, \nu}^{\lambda}\right)^{2} \leq\left(\begin{array}{l}
n \\
k
\end{array}\right) .
$$

Proof. We have:

$$
\begin{gathered}
\sum_{\lambda \vdash n}\left(c_{\mu, \nu}^{\lambda}\right)^{2} \leq \sum_{\lambda \vdash n} c_{\mu, \nu}^{\lambda} \frac{f^{\lambda}}{f^{\mu} f^{\nu}}=\frac{1}{f^{\mu} f^{\nu}} \cdot f^{\mu} f^{\nu}\left(\begin{array}{l}
n \\
k
\end{array}\right)=\left(\begin{array}{l}
n \\
k
\end{array}\right), \\
\sum_{\mu \vdash k, \nu \vdash n-k}\left(c_{\mu, \nu}^{\lambda}\right)^{2} \leq \sum_{\mu \vdash k, \nu \vdash n-k} c_{\mu, \nu}^{\lambda} \frac{f^{\mu} f^{\nu}\left(\begin{array}{l}
n \\
k
\end{array}\right)}{f^{\lambda}}=\frac{1}{f^{\lambda}} \cdot f^{\lambda}\left(\begin{array}{l}
n \\
k
\end{array}\right)=\left(\begin{array}{l}
n \\
k
\end{array}\right),
\end{gathered}
$$

where were repeatedly use both equations in (4.1). 
Corollary 4.3. For every $0 \leq k \leq n$, we have:

$$
\left(\begin{array}{l}
n \\
k
\end{array}\right) \leq \sum_{\lambda \vdash n} \sum_{\mu \vdash k} \sum_{\nu \vdash n-k}\left(c_{\mu, \nu}^{\lambda}\right)^{2} \leq\left(\begin{array}{l}
n \\
k
\end{array}\right) p(n) .
$$

Note that $p(k) p(n-k) \geq p(n)$ (cf. [DP]), which is why we used here only the first inequality from Lemma 4.2 .

4.2. Largest Littlewood-Richardson coefficients. Here we prove theorems [1.5] and [1.6 Proposition 4.4. We have:

$$
\frac{1}{\sqrt{p(k) p(n-k) p(n)}}\left(\begin{array}{l}
n \\
k
\end{array}\right)^{1 / 2} \leq \mathbf{C}(n, k) \leq\left(\begin{array}{l}
n \\
k
\end{array}\right)^{1 / 2} .
$$

Proof. The lower bound follows immediately from Lemma 4.1, while the upper bound follows from Lemma 4.2 .

Theorem 1.5 follows immediately from here:

Corollary 4.5. We have:

$$
\left(\begin{array}{l}
n \\
k
\end{array}\right)^{1 / 2} e^{-d \sqrt{n}(1+o(1))} \leq \mathbf{C}(n, k) \leq\left(\begin{array}{l}
n \\
k
\end{array}\right)^{1 / 2}
$$

where $d=\pi(1+\sqrt{2}) / \sqrt{6} \approx 3.0963$

Proof. Recall that $\log p(n) \sim 2 c_{1} \sqrt{n}$, where $c_{1}=\pi / \sqrt{6}$ defined in (2.5). By log-concavity of the partition function [DP], we also have:

$$
\log [p(k) p(n-k)] \leq 2 \log p(n / 2) \sim 2 \sqrt{2} c_{1} \sqrt{n} .
$$

Now Proposition 4.4 implies the result.

Theorem 4.6. Let $0 \leq k \leq n$ and $\lambda \vdash n$. Suppose $f^{\mu} \geq \sqrt{k !} / a$ and $f^{\nu} \geq \sqrt{(n-k) !} / a$, for some $a \geq 1$. Then there exists $\lambda \vdash n$, such that:

$$
f^{\lambda} \geq \frac{\sqrt{n !}}{a^{2} p(n)}, \quad \text { and } \quad c_{\mu, \nu}^{\lambda} \geq \frac{\sqrt{\left(\begin{array}{l}
n \\
k
\end{array}\right)}}{a^{2} p(n)} .
$$

Proof. Let $\lambda$ be the index of the largest term in the RHS of

$$
\frac{n !}{a^{2} \sqrt{k !(n-k) !}} \leq f^{\mu} f^{\nu}\left(\begin{array}{l}
n \\
k
\end{array}\right)=\sum_{\lambda \vdash k} c_{\mu, \nu}^{\lambda} f^{\lambda} .
$$

On the one hand, by the upper bound in Proposition 4.4 we have:

$$
f^{\lambda} \geq \frac{f^{\mu} f^{\nu}\left(\begin{array}{l}
n \\
k
\end{array}\right)}{p(n) \cdot \mathbf{C}(n, k)} \geq \frac{n !}{a^{2} \sqrt{k !(n-k) !} \cdot p(n) \sqrt{\left(\begin{array}{l}
n \\
k
\end{array}\right)}}=\frac{\sqrt{n !}}{a^{2} p(n)} .
$$

Similarly,

$$
c_{\mu, \nu}^{\lambda} \geq \frac{f^{\mu} f^{\nu}\left(\begin{array}{l}
n \\
k
\end{array}\right)}{p(n) \cdot \mathbf{D}(n)} \geq \frac{n !}{a^{2} \sqrt{k !(n-k) !} \cdot p(n) \sqrt{n !}}=\frac{\sqrt{\left(\begin{array}{l}
n \\
k
\end{array}\right)}}{a^{2} p(n)},
$$

as desired. 
Theorem 4.7. Let $0 \leq k \leq n$ and $\lambda \vdash n$. Suppose $f^{\lambda} \geq \sqrt{n !} / a$, for some $a \geq 1$. Then there exists $\mu \vdash k$ and $\nu \vdash(n-k)$, such that:

$$
f^{\mu} \geq \frac{\sqrt{k !}}{a p(k) p(n-k)}, \quad f^{\nu} \geq \frac{\sqrt{(n-k) !}}{a p(k) p(n-k)} \quad \text { and } \quad c_{\mu, \nu}^{\lambda} \geq \frac{\sqrt{\left(\begin{array}{l}
n \\
k
\end{array}\right)}}{a p(k) p(n-k)} .
$$

Proof. Let $(\mu, \nu)$ be the index of the largest term in the RHS of

$$
\sqrt{n !} / a \leq f^{\lambda}=\sum_{\mu \vdash k} \sum_{\nu \vdash n-k} c_{\mu, \nu}^{\lambda} f^{\mu} f^{\nu} .
$$

On the one hand, by the upper bound in Proposition 4.4 we have:

$$
f^{\mu} f^{\nu} \geq \frac{f^{\lambda}}{p(k) p(n-k) \cdot \mathbf{C}(n, k)} \geq \frac{\sqrt{n !} / a}{p(k) p(n-k) \cdot \sqrt{\left(\begin{array}{l}
n \\
k
\end{array}\right)}}=\frac{\sqrt{k !(n-k) !}}{a p(k) p(n-k)} .
$$

Dividing both sides by $f^{\mu}$ and $f^{\nu}$ gives the first two bounds. Similarly,

$$
c_{\mu, \nu}^{\lambda} \geq \frac{f^{\lambda}}{p(k) p(n-k) \cdot f^{\mu} f^{\nu}} \geq \frac{\sqrt{n !} / a}{p(k) p(n-k) \cdot \sqrt{k !(n-k) !}}=\frac{\sqrt{\left(\begin{array}{l}
n \\
k
\end{array}\right)}}{a p(k) p(n-k)},
$$

as desired.

Theorems 4.6 and 4.7 immediately imply parts $(i)$ and $(i i)$ of Theorem 1.6. They are patterned after Theorem 3.4. Proof of part (iii) uses a combination of different tools and is postponed until 6.4 .

4.3. Stanley's formula. Here we use Lemma 4.1 to obtain the LR-analogue of (3.5) due to Stanley, as his proof is unpublished. First, we need the following recent identity of Harris and Willenbring:

Lemma $4.8([\mathrm{HW}])$. We have:

$$
\sum_{\lambda, \mu, \nu \vdash n}\left(c_{\mu, \nu}^{\lambda}\right)^{2} q^{|\mu|} t^{|\nu|}=\prod_{i=1}^{\infty} \frac{1}{1-q^{i}-t^{i}} .
$$

Proof. Our proof is built on the proof of Lemma 4.1, Recall that

$$
z_{\alpha}:=\prod_{i} i^{m_{i}(\alpha)} m_{i}(\alpha) !
$$

for all $\alpha \vdash n$, where $m_{i}(\alpha)$ denote the number of parts of size $i$ in $\alpha$. We then have:

$$
\begin{aligned}
\sum_{\lambda \vdash n, \mu \vdash k, \nu \vdash n-k}\left(c_{\mu, \nu}^{\lambda}\right)^{2}=\sum_{\alpha \vdash k, \beta \vdash n-k} \frac{z_{\alpha \cup \beta}}{z_{\alpha} \cdot z_{\beta}} & =\sum_{\alpha \vdash k, \beta \vdash n-k} \prod_{i \geq 1} \frac{i^{m_{i}(\alpha \cup \beta)} m_{i}(\alpha \cup \beta) !}{i^{m_{i}(\alpha)} m_{i}(\alpha) ! \cdot i^{m_{i}(\beta)} m_{i}(\beta) !} \\
=\sum_{\alpha \vdash k, \beta \vdash n-k} \prod_{i \geq 1}\left(\begin{array}{c}
m_{i}(\alpha)+m_{i}(\beta) \\
m_{i}(\alpha)
\end{array}\right) & =\left[q^{k} t^{n-k}\right] \prod_{i \geq 1} \frac{1}{1-q^{i}-t^{i}} .
\end{aligned}
$$

Note that the last line follows from $m_{i}(\alpha \cup \beta)=m_{i}(\alpha)+m_{i}(\beta)$. 
Corollary 4.9 (Stanley [S5, Exc. 7.79]).

$$
\sum_{\lambda, \mu, \nu \vdash n}\left(c_{\mu, \nu}^{\lambda}\right)^{2} \sim K 2^{n} \quad \text { as } n \rightarrow \infty,
$$

where

$$
K=\prod_{i=1}^{\infty}\left(1-\frac{1}{2^{i}}\right)^{-1} \approx 3.4627466195
$$

Note that this asymptotic bound implies part $(* *)$ of Stanley's theorem 1.1, On the other hand, neither Theorem 4.7 implies the corollary, nor vice versa.

Proof of Corollary [4.9. Let $p_{2}(n)$ is the number of bicolored partitions of $n$ defined as follows (see [OEIS, A070933]) :

$$
\sum_{n=0}^{\infty} p_{2}(n) t^{n}=\prod_{i=1}^{\infty} \frac{1}{1-2 t^{i}}
$$

and recall that $p_{2}(n) \sim K 2^{n}$. Taking $q=t$ in Lemma 4.8, we obtain the result.

4.4. Largest refined LR-coefficients. By analogy with the Kronecker coefficients, define

$$
\mathbf{C}(\lambda):=\max _{0 \leq k \leq n} \max _{\mu \vdash k} \max _{\nu \vdash n-k} c_{\mu, \nu}^{\lambda} .
$$

Theorem 4.10. Fix $\lambda \vdash n$ and let $\ell \geq \ell(\lambda)$. Then:

$$
\mathbf{C}(\lambda)^{2} \leq \prod_{(i, j) \in \lambda} \frac{2 \ell+j-i}{\ell+j-i}
$$

Letting $\ell \rightarrow \infty$ gives Stanley's upper bound $\mathbf{C}(\lambda) \leq 2^{n / 2}$, but gives a much better bound for smaller $\ell$ (see below).

Proof. We have:

$$
\begin{aligned}
s_{\lambda}\left(1^{2 \ell}\right) & =\sum_{\mu, \nu} c_{\mu, \nu}^{\lambda} s_{\mu}\left(1^{\ell}\right) s_{\nu}\left(1^{\ell}\right)=\sum_{\rho, \mu, \nu} c_{\mu, \nu}^{\lambda} c_{\mu, \nu}^{\rho} s_{\rho}\left(1^{\ell}\right) \\
& \geq s_{\lambda}\left(1^{\ell}\right) \sum_{\mu, \nu}\left(c_{\mu, \nu}^{\lambda}\right)^{2} \geq s_{\lambda}\left(1^{\ell}\right) \mathbf{C}(\lambda)^{2} .
\end{aligned}
$$

By the hook-content formula (2.3), we conclude:

$$
\mathbf{C}(\lambda)^{2} \leq \frac{s_{\lambda}\left(1^{2 \ell}\right)}{s_{\lambda}\left(1^{\ell}\right)}=\left[\prod_{(i, j) \in \lambda} \frac{2 \ell+j-i}{h_{i j}}\right] \cdot\left[\prod_{(i, j) \in \lambda} \frac{\ell+j-i}{h_{i j}}\right]^{-1}=\prod_{(i, j) \in \lambda} \frac{2 \ell+j-i}{\ell+j-i}
$$

as desired.

Corollary 4.11. For all $\lambda \vdash n$ and $\ell=\ell(\lambda), m=\lambda_{1}$ we have:

$$
\mathbf{C}(\lambda) \leq(m+\ell)^{\ell^{2} / 2} .
$$


Proof. For a fixed $\ell=\ell(\lambda)$ and $n=|\lambda|$, the product in (4.4) maximizes when $m=\lambda_{1}=\lceil n / \ell\rceil$. Indeed, moving the squares below and to the left decreases the contents $(j-i)$, and thus increases the ratios $(2 \ell+j-i) /(\ell+j-i)$ in the product. Thus, we have:

$$
\mathbf{C}(\lambda)^{2} \leq \prod_{i=1}^{\ell} \prod_{j=1}^{m} \frac{2 \ell+j-i}{\ell+j-i} \leq \prod_{i=1}^{\ell} \prod_{j=m-\ell+1}^{m}(2 \ell+j-i) \leq(m+\ell)^{\ell^{2}} .
$$

This implies the result.

Remark 4.12. It follows from the proof that the RHS of (4.4) maximizes at a rectangle $\lambda=$ $m^{\ell}$ (cf. [BG]). Note, however, that $c_{\mu, \nu}^{\lambda} \leq 1$ in this case, see e.g. [PP1, §4.3].

\subsection{Largest $\mathbf{L R}-$ coefficient with few rows. Define}

$$
\mathbf{C}_{\ell}(n):=\max _{\lambda \vdash n, \ell(\lambda)=\ell} \max _{\mu \vdash k} \max _{\nu \vdash n-k} c_{\mu, \nu}^{\lambda} .
$$

Theorem 4.13. For all $\ell, n \geq 1$, we have:

$$
n^{\frac{1}{2} \ell^{2}-a \ell} e^{-b \ell^{2} \log \ell} \leq \mathbf{C}_{\ell}(n) \leq(n+1)^{\frac{1}{2} \ell^{2}},
$$

for some universal constants $a, b>0$.

Corollary 4.14. Let $\left\{\ell_{n}\right\}$ be an integer sequence which satisfies $\ell_{n}=O(\sqrt{n} / \log n)$ and $\ell_{n}=\omega(1)$. Then we have:

$$
\log \mathbf{C}_{\ell_{n}}(n) \sim \frac{1}{2}\left(\ell_{n}\right)^{2} \log n
$$

Proof of Theorem 4.13. The upper bound follows from Corollary 4.11 since $m+\ell \leq n+1$. The following argument is more direct and gives a slightly better bound; we include it for completeness.

Recall the combinatorial interpretation for $c_{\mu, \nu}^{\lambda}$ in terms of the number of Knutson-Tao hives [KT1] (see also [PV] and BZ-patterns in [S4, §7.A1.3]). These are integer triangular tables of size $(\ell+1)$, with entries $\leq n$ and fixed boundary given by $\lambda, \mu$ and $\nu$. This leaves only $\left(\begin{array}{c}\ell-1 \\ 2\end{array}\right)$ entries, which immediately implies the upper bound

$$
\mathbf{C}_{\ell}(n) \leq(n+1)^{(\ell-1)(\ell-2) / 2} .
$$

For the lower bound, recall the combinatorial interpretations for $c_{\mu, \nu}^{\lambda}$ in terms of honeycombs [KT1] and in terms of the Knutson-Tao puzzles [KT2]. The latter are triangles of size $(m+\ell)$, where $m=\lambda_{1}$, which are tiled with pieces of 3 different types (up to rotation and parallel translation). Note that these puzzles are uniquely determined by the positions of the (111)-triangles, since the lozenges extend the rows of 1-s between them and (000)-triangles fill the remaining space.

Consider the honeycomb graph of 1-edges in the graph $\Gamma$ dual to the puzzle graph. Fix $\Gamma$ with $r=\left(\begin{array}{l}\ell \\ 2\end{array}\right)$ regular hexagons of size $m /(3 \ell)$. Let the faces of $\Gamma$ "breathe", i.e. contract by at most $m /(18 \ell)$. The changes to honeycombs are small enough, so that none of them degenerate (since none of the edges degenerate). Pin one of the central vertices of the honecomb at a

fixed position in the center of the triangle. All honeycombs will fit the $(m+\ell)$ triangle, and the resulting puzzles are distinct then. Their number is at least

$$
\left(\frac{m}{18 \ell}\right)^{r} \geq\left(\frac{n}{18 \ell^{2}}\right)^{r}
$$


Since the total number of triples of partitions $(\lambda, \mu, \nu)$ is at most

$$
\left(\begin{array}{l}
n \\
\ell
\end{array}\right)^{3} \leq n^{3 \ell},
$$

at least one triple has $\geq n^{r-3 \ell}\left(18 \ell^{2}\right)^{-r}$ puzzles, as desired.

4.6. Vanishing LR-coefficients. We believe the natural analogue of Conjecture 3.10 fails for the LR-coefficients.

Conjecture 4.15. There exists three Plancherel partitions sequences $\left\{\lambda^{(n)} \vdash n\right\},\left\{\mu^{(n)} \vdash n / 2\right\}$ and $\left\{\nu^{(n)} \vdash n / 2\right\}$, s.t.

$$
\frac{1}{\sqrt{n}}\left(\frac{n}{2}-\log _{2} c_{\mu^{(n)}, \nu^{(n)}}^{\lambda^{(n)}}\right) \rightarrow \infty
$$

The following result gives a strong evidence in favor of the conjecture.

Proposition 4.16. Let $\left\{\mu^{(n)} \vdash n / 2\right\},\left\{\nu^{(n)} \vdash n / 2\right\}$ be two Plancherel partitions sequences. Then there exist a partition sequence $\left\{\lambda^{(n)} \vdash n\right\}$ with

$$
f^{\lambda^{(n)}}=\sqrt{n !} e^{O(\sqrt{n} \log n)}
$$

such that:

$$
c_{\mu^{(n)}, \nu^{(n)}}^{\lambda^{(n)}}=0 .
$$

The proposition should be compared with part $(i)$ of Theorem 1.6. The notable difference in the statement is the $(\log n)$ factor in the error term.

Proof. For a partition $\lambda=\left(\lambda_{1}, \lambda_{2}, \ldots\right) \vdash n$, let

$$
\widehat{\lambda}:=\left(\lambda_{3}, \lambda_{4}, \ldots\right) \cup 1^{\lambda_{1}+\lambda_{2}}
$$

Fix a Plancherel partition sequence $\left\{\lambda^{(n)}\right\}$. Then $\left\{\widehat{\lambda}^{(n)}\right\}$ satisfies (4.5), and has $\ell\left(\lambda^{(n)}\right) \sim 6 \sqrt{n}$ parts. Since $\left\{\mu^{(n)}\right\},\left\{\nu^{(n)}\right\}$ are Plancherel, they have $2 \sqrt{n}(1+o(1))$ parts. Since $\left\{\mu^{(n)} \circ \nu^{(n)}\right\}$ have at most $4 \sqrt{n}(1+o(1))$ parts, this implies the result.

4.7. Containment of the largest LR-coefficients. We start with the following beautiful result by Lam, Postnikov and Pylyavskyy [LPP] which we state in the following equivalent form.

Theorem 4.17 ([LPP]). Let $\lambda \vdash n, \mu \vdash k, \nu \vdash n-k$. Denote $\alpha=\mu \cap \nu, \beta=\mu \cup \nu$. Then

$$
c_{\mu, \nu}^{\lambda} \leq c_{\alpha, \beta}^{\lambda} .
$$

This implies that the largest LR-coefficient is attained at a flag of partitions.

Corollary 4.18. Let $\lambda \vdash n$. There exist $\alpha \subseteq \beta \subset \lambda,|\alpha|+|\beta|=n$, s.t.

$$
\mathbf{C}(\lambda)=c_{\alpha, \beta}^{\lambda} \text {. }
$$

In particular, for every $n$, there exist $\alpha \subseteq \beta \subset \lambda,|\alpha|+|\beta|=|\lambda|=n$, s.t.

$$
\mathbf{C}(n)=c_{\alpha, \beta}^{\lambda} \text {. }
$$


Remark 4.19. Note that for some $n \geq 10$, the sequence $\{\mathbf{C}(n, k), 0 \leq k \leq n\}$ is not unimodal (see A). Corollary 4.18 suggests an explanation why not: the unimodality would imply that for $k=n / 2$ we have $\alpha=\beta$. Denote by $\zeta(n)$ the smallest $k$ s.t. $\mathbf{C}(n, k)=\mathbf{C}(n)$. Let $\rho(n):=n / 2-\zeta(n)$. For example, $\zeta(18)=7$ since $\mathbf{C}(18,7)=\mathbf{C}(18)=11$, so $\rho(18)=2$.

What can be said about the asymptotics of $\rho(n)$ ? Theorem 1.5 implies that $\rho(n)=o(n)$. It would be interesting to find a nontrivial lower bound on $\rho(n)$. Finally, we make the following conjecture based on our computer experiments.

Conjecture 4.20. Let $c_{\mu, \nu}^{\lambda}=\mathbf{C}(n)$, where $\lambda \vdash n$. Then $\mu \subseteq \nu$ or $\nu \subseteq \mu$.

In other words, we are claiming that "there exist" clause in the corollary can be replaced with "for all". For example, for $n=18$ the maximum $\mathbf{C}(18)=11$ is attained at $\lambda=75321$, $\mu=5321$ and $\nu=421$ and its conjugates and transpositions:

$$
c_{\mu, \nu}^{\lambda}=c_{\nu, \mu}^{\lambda}=c_{\mu^{\prime}, \nu^{\prime}}^{\lambda^{\prime}}=c_{\nu^{\prime}, \mu^{\prime}}^{\lambda^{\prime}}=11 .
$$

Here $\lambda \supset \mu \supset \nu$.

4.8. Monotonicity and stability of the largest LR-coefficients. We conclude this section with the following results suggested by numerical values for $\mathbf{C}(n, k)$ in the appendix $\AA$ A.

Proposition 4.21. The sequence $\{\mathbf{C}(n)\}$ is nondecreasing. Similarly, for every fixed $k \geq 1$, the sequence $\{\mathbf{C}(n, k)\}$ is bounded and nondecreasing. In particular, for every fixed $k$, the sequences $\{\mathbf{C}(n, k)\}$ stabilizes when $n$ is large enough.

Proof. Recall the usual Young tableaux combinatorial interpretation of the LR-coefficients, see e.g. [S4]. It is well known and easy to see that

$$
c_{\mu, \nu}^{\lambda} \leq c_{\mu, \nu+1}^{\lambda+1},
$$

where $\left(\lambda_{1}, \lambda_{2}, \lambda_{3}, \ldots\right)+1:=\left(\lambda_{1}+1, \lambda_{2}, \lambda_{3}, \ldots\right)$. By fixing $\mu$, we conclude that $\mathbf{C}(n) \leq \mathbf{C}(n+1)$ and $\mathbf{C}(n, k) \leq \mathbf{C}(n, k+1)$, for all $n \geq 1, k \geq 0$.

To prove that $\mathbf{C}(k, k), \mathbf{C}(k+1, k), \mathbf{C}(k+2, k), \ldots$ is bounded, observe that

$$
c_{\mu, \nu}^{\lambda} \leq \frac{f^{\lambda / \nu}}{f^{\mu}} \leq k !
$$

for all $\mu \vdash k$. This implies the result.

Theorem 4.22. For every $k \geq 0$ and $n \geq\left(\begin{array}{c}k+1 \\ 2\end{array}\right)$, we have $\mathbf{C}(n, k)=\mathbf{D}(k)$.

Proof. By Proposition 4.21, we have $\mathbf{C}(k, k) \leq \mathbf{C}(k+1, k) \leq \mathbf{C}(k+2, k) \leq \ldots$ Recall that the $S_{k^{-m o d u l e}} \mathbb{S}^{\lambda / \nu}$ corresponding to skew shape $\lambda / \nu$ is a submodule of the regular representation (see e.g. $\underline{\mathrm{Sag}}$ ). Equivalently,

$$
\left(e_{1}\right)^{n}-s_{\lambda / \mu} \geq 0
$$

is Schur positive (cf. [BBR, $[\mathrm{LPP}]$ ). This implies that $c_{\mu, \nu}^{\lambda} \leq f^{\nu}$. We conclude that $\mathbf{C}(n, k) \leq$ $\mathbf{D}(k)$ for all $n \geq k$.

Denote $\delta_{k}=(k-1, k-2, \ldots, 1)$ and let $r=n-\left(\begin{array}{c}k+1 \\ 2\end{array}\right)$. Define $\lambda=\delta_{k+1}+(r), \nu=\delta_{k}+(r)$, and observe that $\lambda / \nu$ is a disjoint union of $k$ squares. Then $\mathbb{S}^{\lambda / \nu}$ is regular and $\mathbf{C}(n, k)=\mathbf{D}(k)$ for all $n \geq\left(\begin{array}{c}k+1 \\ 2\end{array}\right)$. 
Remark 4.23. These results on stability of the largest LR-coefficients are modeled after similar results on stability of certain Kronecker coefficients (see e.g. [BOR, Man, SS2]). Of course, the latter results are more technical since there is no combinatorial interpretation for Kronecker coefficients.

By the theorem, $\{\mathbf{C}(n, k)\}$ stabilizes at or before $\left(\begin{array}{c}k+1 \\ 2\end{array}\right)$. The data in the appendix for $0 \leq k \leq 6$, suggests that it stabilizes precisely at $\left(\begin{array}{c}k+1 \\ 2\end{array}\right)$. We conjecture that this is true for all $k \geq 0$.

\section{LARGE LR-COEFFICIENTS IMPLY LARGE DIMENSIONS}

The goal of this section is obtain Theorem 5.3, which is the LR-analogue of the bound $f^{\lambda} \geq g(\lambda, \mu, \nu)$ in (3.2).

5.1. Upper bounds. To simplify presentation, here and everywhere in this section we use (finite) sums over partitions for which the corresponding terms are well-defined and nonzero.

Lemma 5.1. Let $\mu \vdash k$ and $\nu \vdash(n-k)$. Then

$$
\sum_{\lambda \vdash n}\left(c_{\mu, \nu}^{\lambda}\right)^{2}=\sum_{\alpha, \beta, \gamma, \delta} c_{\alpha, \gamma}^{\mu} c_{\alpha, \delta}^{\mu} c_{\beta, \gamma}^{\nu} c_{\beta, \delta}^{\nu} .
$$

Proof. Let $\mu, \nu, \tau, \rho$ be fixed partitions. Start with the skew Cauchy identity for Schur functions:

$$
\sum_{\lambda} s_{\lambda / \mu}(\mathbf{x}) s_{\lambda / \tau}(\mathbf{y})=\prod_{i=1}^{\infty} \prod_{j=1}^{\infty} \frac{1}{1-x_{i} y_{j}} \sum_{\alpha} s_{\tau / \alpha}(\mathbf{x}) s_{\mu / \alpha}(\mathbf{y}) .
$$

(see e.g. [SS1]). We expand it as follows:

$$
\begin{aligned}
\sum_{\lambda, \nu, \tau} c_{\mu, \nu}^{\lambda} s_{\nu}(\mathbf{x}) c_{\tau, \rho}^{\lambda} s_{\rho}(\mathbf{y}) & =\sum_{\beta} s_{\beta}(\mathbf{x}) s_{\beta}(\mathbf{y}) \sum_{\alpha, \gamma, \delta} c_{\alpha, \gamma}^{\tau} s_{\gamma}(\mathbf{x}) c_{\alpha, \delta}^{\mu} s_{\delta}(\mathbf{y}) \\
& =\sum_{\alpha, \beta, \gamma, \delta, \nu, \tau} c_{\alpha, \gamma}^{\tau} c_{\alpha, \delta}^{\mu} c_{\beta, \gamma}^{\nu} c_{\beta, \delta}^{\rho} s_{\nu}(\mathbf{x}) s_{\rho}(\mathbf{y}) .
\end{aligned}
$$

The needed identity follows then by taking the coefficients at $s_{\nu}(\mathbf{x}) s_{\rho}(\mathbf{y})$ from both sides:

$$
\sum_{\lambda} c_{\mu, \nu}^{\lambda} c_{\tau, \rho}^{\lambda}=\sum_{\alpha, \beta, \gamma, \delta} c_{\alpha, \gamma}^{\tau} c_{\alpha, \delta}^{\mu} c_{\beta, \gamma}^{\nu} c_{\beta, \delta}^{\rho}
$$

Taking $\tau=\mu$ and $\rho=\nu$ implies the result.

Lemma 5.2. Let $\lambda \vdash n, \mu \vdash k$ and $\nu \vdash(n-k)$. Then:

$$
c_{\mu, \nu}^{\lambda} \leq \sqrt{n} p(k) p(n-k) \mathbf{C}(\mu) \mathbf{C}(\nu) .
$$

Proof. We have:

$$
\begin{aligned}
\left(c_{\mu, \nu}^{\lambda}\right)^{2} & \leq \sum_{\lambda \vdash n}\left(c_{\mu, \nu}^{\lambda}\right)^{2}=\sum_{\alpha, \beta, \gamma, \delta} c_{\alpha, \gamma}^{\mu} c_{\alpha, \delta}^{\mu} c_{\beta, \gamma}^{\nu} c_{\beta, \delta}^{\nu} \\
& \leq \sum_{a=0}^{\min \{k, n-k\}} p(k-a) p(n-k-a) p(a)^{2} \mathbf{C}(\mu)^{2} \mathbf{C}(\nu)^{2} \\
& \leq n p(k)^{2} p(n-k)^{2} \mathbf{C}(\mu)^{2} \mathbf{C}(\nu)^{2}
\end{aligned}
$$

which implies the result. 
Theorem 5.3. Let $\lambda \vdash n,|\mu|+|\nu|=n$ be fixed partitions. Then:

$$
f^{\lambda} \geq e^{-u n}\left(c_{\mu, \nu}^{\lambda}\right)^{\log _{2} n}
$$

where $u>0$ is a universal constant.

Proof. The idea is to iteratively apply the inequalities

$$
f^{\lambda} \geq f^{\mu} f^{\nu} c_{\mu, \nu}^{\lambda}
$$

and the following corollary of Lemma 5.2 .

$$
c_{\mu, \nu}^{\lambda} \leq e^{a \sqrt{n}} \mathbf{C}(\mu) \mathbf{C}(\nu),
$$

for some universal constant $a>0$.

Consider a full binary tree $T=T(\lambda, \mu, \nu)$ of depth $m=\left\lceil\log _{2} n\right\rceil$ defined as follows. Let the nodes be labeled by partitions according to the following rule:

(i) $\lambda$ is the root with children $\mu$ and $\nu$;

(ii) every non-leaf node $\rho$ has children $\alpha, \beta$ so that $c_{\alpha, \beta}^{\rho}=\max _{\phi, \psi} c_{\phi, \psi}^{\rho}$

(these could be empty partitions).

For each node $\rho \in T$, denote by $L(\rho)$ and $R(\rho)$ its children in $T$, and let $k_{\rho}:=|\rho|$ be the size of the partition $\rho$. Iterating the inequality (5.2) along $T$ starting from the root $\lambda$ we obtain:

$$
f^{\lambda} \geq f^{L(\lambda)} f^{R(\lambda)} c_{L(\lambda), R(\lambda)}^{\lambda} \geq \cdots \geq \prod_{\rho \in T} c_{L(\rho), R(\rho)}^{\rho} .
$$

From (5.3) we have the following local inequalities:

$$
c_{L(\alpha), R(\alpha)}^{\alpha} c_{L(\beta), R(\beta)}^{\beta} \geq e^{-a \sqrt{k_{\rho}}} c_{\alpha, \beta}^{\rho}, \quad \text { where } \rho \in T, \rho \vdash k_{\rho}, \alpha=L(\rho), \beta=R(\rho) .
$$

Applying these inequalities in (5.4) repeatedly, starting from the bottom $(m-1)$-st level to top (the root at 0 -th level), we obtain:

$$
f^{\lambda} \geq \prod_{\rho \in T} c_{L(\rho), R(\rho)}^{\rho} \geq\left[\prod_{i=0}^{m-1} \prod_{\rho \in T_{i}} e^{-a \sqrt{k_{\rho}}(m-1-i)}\right]\left(c_{\mu, \nu}^{\lambda}\right)^{m},
$$

where $T_{i}$ denotes the $i$-th level of $T$. By construction, we have $\sum_{\rho \in T_{i}} k_{\rho}=n$ for every level $0 \leq i \leq m$, with $\left|T_{i}\right|=2^{i}$. Using the Cauchy-Schwarz inequality we get

$$
\sum_{\rho \in T_{i}} \sqrt{k_{\rho}} \leq \sqrt{2^{i} \sum_{\rho \in T_{i}} k_{\rho}}=\sqrt{2^{i}} \sqrt{n} .
$$

This implies

$$
\prod_{\rho \in T_{i}} e^{-a \sqrt{k_{\rho}}(m-1-i)} \geq e^{-a \sqrt{n} \sqrt{2^{i}}(m-1-i)} .
$$

Note that

$$
\sum_{i=0}^{m-1} \sqrt{2^{i}}(m-1-i)=(3+2 \sqrt{2})\left(\sqrt{2^{m}}-1\right)-m(1+\sqrt{2})=O\left(\sqrt{2^{m}}\right)=O(\sqrt{n}) .
$$

From here and (5.5), we conclude that

$$
f^{\lambda} \geq \exp \left(-a \sqrt{n} \sum_{i=0}^{m-1} \sqrt{2^{i}}(m-1-i)\right)\left(c_{\mu, \nu}^{\lambda}\right)^{m} \geq e^{-u n}\left(c_{\mu, \nu}^{\lambda}\right)^{\log _{2} n},
$$


for some universal constant $u \geq 0$, as desired.

5.2. Proof of Theorem 1.7. By assumption, $k_{n}=\lfloor n / 2\rfloor$. From the inequality (5.1) we conclude that

$$
\log f^{\lambda^{(n)}} \geq\left(\log _{2} n\right) \cdot \log \left[2^{n / 2} e^{O(n / \log n)}\right]-O(n)=\frac{1}{2} n \log n-O(n) .
$$

Using the inequality

$$
f^{\nu} \geq \frac{f^{\lambda} c_{\mu \nu}^{\lambda}}{f^{\mu}\left(\begin{array}{l}
n \\
k
\end{array}\right)}
$$

we obtain:

$$
\begin{aligned}
\log f^{\nu^{(n)}} \geq & \log f^{\lambda^{(n)}}+\log c_{\mu^{(n)}, \nu^{(n)}}^{\lambda^{(n)}}-\log f^{\mu^{(n)}}-\log \left(\begin{array}{c}
n \\
k_{n}
\end{array}\right) \\
\geq & \frac{1}{2} n \log n-O(n)+\log \left(\begin{array}{c}
n \\
n / 2
\end{array}\right)-O(n / \log n) \\
& \quad-\frac{1}{2}(n / 2) \log (n / 2)+O(n)-\log \left(\begin{array}{c}
n \\
n / 2
\end{array}\right) \\
\geq & \frac{1}{2}(n / 2) \log (n / 2)-O(n)=\log \sqrt{(n / 2) !}-O(n) .
\end{aligned}
$$

The inequality for $f^{\mu^{(n)}}$ follows similarly.

Remark 5.4. Stronger versions of the inequality (5.1) would give better bounds for Theorem 1.7. Notably, suppose (5.1) holds with $u=1 / 2$, then

$$
c_{\mu^{(n)}, \nu^{(n)}}^{\lambda^{(n)}}=2^{n / 2} e^{o(n / \log n)}
$$

imply

$$
f^{\lambda^{(n)}} \geq \sqrt{n !} e^{o(n)} \quad \text { and } \quad f^{\mu^{(n)}}, f^{\nu^{(n)}} \geq \sqrt{(n / 2) !} e^{o(n)} .
$$

When $\left\{\lambda^{(n)}\right\},\left\{\mu^{(n)}\right\}$ and $\left\{\nu^{(n)}\right\}$ have strongly stable limit shapes, this would imply that all three partition sequences have VKLS shape by Theorem 2.1.

Conjecture 5.5. Let $\lambda \vdash n$ and $\mu, \nu \vdash n / 2$ be fixed partitions. Let

$$
\widetilde{f}^{\lambda}:=\frac{f^{\lambda}}{\sqrt{n !}} \quad \text { and } \quad \widetilde{c}_{\mu, \nu}^{\lambda}:=\frac{c_{\mu, \nu}^{\lambda}}{\sqrt{\left(\begin{array}{c}
n \\
n / 2
\end{array}\right)}} .
$$

Then:

$$
\tilde{f}^{\lambda} \geq a\left(\widetilde{c}_{\mu, \nu}^{\lambda}\right)^{u \log n},
$$

where $a, u>0$ are universal constants.

This is a strong conjecture. If true, then

$$
c_{\mu^{(n)}, \nu^{(n)}}^{\lambda^{(n)}}=2^{n / 2} e^{O(\sqrt{n})}
$$

imply

$$
f^{\lambda^{(n)}} \geq \sqrt{n !} e^{O(\sqrt{n} \log n)} \quad \text { and } \quad f^{\mu^{(n)}}, f^{\nu^{(n)}} \geq \sqrt{(n / 2) !} e^{O(\sqrt{n} \log n)}
$$




\section{Standard Young tableaux of Skew Shape}

6.1. Sum of squares. We start our study of $f^{\lambda / \mu}=\# \operatorname{SYT}(\lambda / \mu)$ with the following technical result.

Lemma 6.1. For every $0 \leq m \leq n$, we have:

$$
\frac{(n-1) !}{(m-1) !} \leq \sum_{\lambda \vdash n} \sum_{\mu \vdash m}\left(f^{\lambda / \mu}\right)^{2} \leq \frac{n !}{m !} p(m) .
$$

Proof. We start with the following Stanley's identity given in [S1]:

$$
\sum_{m=0}^{\infty} \sum_{n=0}^{\infty} \sum_{\mu \vdash m} \sum_{\lambda \vdash m+n}\left(f^{\lambda / \mu}\right)^{2} \frac{q^{m} t^{n}}{n !}=\frac{1}{1-t /(1-q)} \prod_{i=1}^{\infty} \frac{1}{1-q^{i}} .
$$

Taking the coefficient $\left[q^{m} t^{n-m}\right]$ on both sides gives:

$$
\sum_{\lambda \vdash n} \sum_{\mu \vdash m}\left(f^{\lambda / \mu}\right)^{2}=(n-m) ! \sum_{k=1}^{m}\left(\begin{array}{c}
n-m+k-1 \\
k-1
\end{array}\right) p(m-k) .
$$

Bounding $p(m-k) \leq p(m)$ and summing the binomials give the upper bound:

$$
\sum_{\lambda \vdash n} \sum_{\mu \vdash m}\left(f^{\lambda / \mu}\right)^{2} \leq(n-m) !\left(\begin{array}{c}
n \\
m
\end{array}\right) p(m) .
$$

Finally, the term $k=m$ in the RHS of (6.1) gives the lower bound.

6.2. Largest \# SYTs of skew shape. For $0 \leq m \leq n$, define

$$
\mathrm{F}(m, n):=\max _{\lambda \vdash n} \max _{\mu \vdash m} f^{\lambda / \mu} .
$$

Corollary 6.2. Let $1 \leq m \leq n$, and $n \rightarrow \infty$. Then:

$$
\sqrt{\frac{n !}{m !}} e^{-2 c_{1} \sqrt{n}(1+o(1))} \leq \mathrm{F}(m, n) \leq \sqrt{\frac{n !}{m !}} e^{2 c_{1} \sqrt{n}(1+o(1))} .
$$

Proof. Let $1 \leq m \leq n$. From Lemma 6.1, we immediately have:

$$
\sqrt{\frac{(n-1) !}{(m-1) !}} \frac{1}{\sqrt{p(m) p(n)}} \leq \mathrm{F}(m, n) \leq \sqrt{\frac{n !}{m !}} \sqrt{p(m)} .
$$

These bounds imply the result.

\subsection{Skew shapes with the largest \# SYTs.}

Theorem 6.3. Let $m_{n}:=\lfloor\theta n\rfloor$ for some $0<\theta<1$, and let $\left\{\lambda^{(n)} \vdash n\right\},\left\{\mu^{(n)} \vdash m_{n}\right\}$ be two Plancherel partitions sequences. Then:

$$
f^{\lambda^{(n)} / \mu^{(n)}}=\sqrt{\frac{n !}{m_{n} !}} e^{-O\left(n^{2 / 3} \log n\right)} .
$$


Proof. By Theorem 1.3, we know that $\mu^{(n)}$ is contained in $s \cdot \psi$ of area at most $m_{n}+d n^{2 / 3}$ for some constant $d>0$. Indeed, each of the $O(\sqrt{n})$ rows of $\mu^{(n)}$ differs from the $\sqrt{n} \psi$ by at most $O\left(n^{1 / 6}\right)$. Similarly, every Plancherel $\widetilde{\mu}^{(n)}$ contains $t \cdot \psi$ of area at least $m_{n}-d n^{2 / 3}$.

Let $\widetilde{m}_{n}=m_{n}+2 d n^{2 / 3}$. By Theorem 4.7, for every Plancherel partitions sequence there exists Plancherel sequences $\left\{\widetilde{\mu}^{(n)} \vdash \widetilde{m}_{n}\right\}$ and $\left\{\nu^{(n)} \vdash n-\widetilde{m}_{n}\right\}$, s.t.

$$
c_{\widetilde{\mu}^{(n)}, \nu^{(n)}}^{\lambda^{(n)}}=\left(\begin{array}{c}
n \\
\tilde{m}_{n}
\end{array}\right)^{1 / 2} e^{-O(\sqrt{n})}=\left(\begin{array}{c}
n \\
m_{n}
\end{array}\right)^{1 / 2} e^{-O\left(n^{2 / 3} \log n\right)} .
$$

Note that

$$
f^{\nu^{(n)}}=\sqrt{\left(n-\widetilde{m}_{n}\right) !} e^{-O(\sqrt{n})}=\sqrt{\left(n-m_{n}\right) !} e^{-O\left(n^{2 / 3} \log n\right)} .
$$

From above, $\mu_{n} \subset t \cdot \psi \subset \widetilde{\mu}_{n}$, where $t=\sqrt{\theta n+d n^{2 / 3}}$. Thus:

$$
f^{\lambda^{(n)} / \mu^{(n)}} \geq f^{\lambda^{(n)} / \widetilde{\mu}^{(n)}} \geq c_{\widetilde{\mu}^{(n)}, \nu^{(n)}}^{\lambda^{(n)}} \cdot f^{\nu^{(n)}}=\sqrt{\frac{n !}{m_{n} !}} e^{-O\left(n^{2 / 3} \log n\right)} .
$$

The upper bound follows from Corollary 6.2 .

We also have a partial converse:

Theorem 6.4. Let $m_{n}:=\lfloor\theta n\rfloor$, where $\theta=1 / 2$. Let $\left\{\lambda^{(n)} \vdash n\right\}$ and $\left\{\mu^{(n)} \vdash m_{n}\right\}$ be two partitions sequences which satisfy

$$
f^{\lambda^{(n)} / \mu^{(n)}}=\sqrt{\frac{n !}{(n / 2) !}} e^{O(n / \log n)} .
$$

Then

$$
f^{\lambda^{(n)}}=\sqrt{(n / 2) !} e^{O(n)} \quad \text { and } \quad f^{\mu^{(n)}}=\sqrt{(n / 2) !} e^{O(n)} .
$$

Proof. Recall that

$$
f^{\lambda / \mu}=\sum_{\nu \vdash n-m_{n}} c_{\mu, \nu}^{\lambda} f^{\nu}
$$

(see e.g. [S4]). Thus

$$
c_{\mu, \nu}^{\lambda} \geq \frac{f^{\lambda / \mu}}{p(n) \sqrt{|\lambda / \mu| !}}
$$

for some $\nu \vdash|\lambda / \mu|$. Letting $\lambda \leftarrow \lambda^{(n)}, \mu \leftarrow \mu^{(n)}$ and $\nu \leftarrow \nu^{(n)}$ corresponding to maximal LR-coefficient as above, we conclude that

$$
c_{\mu^{(n)}, \nu^{(n)}}^{\lambda^{(n)}}=\left(\begin{array}{c}
n \\
n / 2
\end{array}\right)^{1 / 2} e^{-O(n / \log n)} .
$$

By Theorem 1.7, this implies the result. 
6.4. Proof of Theorem 1.6. Parts $(i)$ and (ii) are proved in 84.2 , For (iii), start with Theorem 6.3 which gives

$$
f^{\lambda^{(n)} / \mu^{(n)}}=\sqrt{\frac{n !}{m_{n} !}} e^{-O\left(n^{2 / 3} \log n\right)}
$$

Write

$$
f^{\lambda^{(n)} / \mu^{(n)}}=\sum_{\nu \vdash n-m_{n}} c_{\mu^{(n)}, \nu}^{\lambda^{(n)}} f^{\nu}
$$

and let $\left\{\nu^{(n)}\right\}$ be the largest terms in the summation. Since

$$
c_{\mu^{(n)}, \nu^{(n)}}^{\lambda^{(n)}} \leq \sqrt{\left(\begin{array}{c}
n \\
m_{n}
\end{array}\right)} \quad \text { and } \quad f^{\nu} \leq \sqrt{\left(n-m_{n}\right) !},
$$

we can follow the proof of Theorem 4.6 to obtain the result.

\section{Final REMARKS AND OPEN PROBlems}

7.1. There is a large body of work on Littlewood-Richardson coefficients and its generalizations, but there are very few explicit formulas and general inequalities. Even when such inequalities exist, they are difficult and not very sharp see e.g. [BBR, $\mathrm{CL}, \mathrm{LPP}$. The exact evaluations are even more rare and use ad hoc methods, see e.g. [CDW]. A notable sharp lower bound in $[\mathrm{Nar}]$ applies only to partitions with the smallest part $\lambda_{\ell} \geq \ell^{2}$.

For Kronecker coefficients, there are even fewer bounds, so much that even positivity is known in very few cases, see [PP2] for an overview. We should note a strongly related work by Biane [Bia], who discusses the limit shape of the random Kronecker and LR-coefficients weighted by the dimensions $f^{\lambda}$.

Finally, despite the Feit determinant formula for $f^{\lambda / \mu}$, getting good (theoretical) asymptotic estimates for the number of standard Young tableaux of skew shape remains difficult. We refer to a survey article [AR] for exact formulas (cf. [MPP2]), and to [MPP1] for the upper and lower bounds.

7.2. Most results in the paper on Kronecker coefficients extend directly to all finite groups with few conjugacy classes. This is a byproduct of the lack of available tools for Kronecker coefficients. On the other hand, the LR-coefficients correspond to induced coefficients with certain additional properties. Notably, the results of Section 5 do not extend to general groups, and dimensions of skew shape representations discussed in Section 6 cannot even be formulated.

7.3. A direct combinatorial proof of Theorem 4.17 would give a combinatorial interpretation of the difference of LR-coefficients as in the theorem. If such a combinatorial interpretation is found, it would perhaps help establish Conjecture 4.20.

7.4. Let us highlight one minor result:

$$
c_{\mu, \nu}^{\lambda} \leq \sqrt{\left(\begin{array}{l}
n \\
k
\end{array}\right)} \quad \text { for all } \quad \lambda \vdash n, \mu \vdash k, \nu \vdash n-k
$$

(see Lemma 4.1). This bound is very natural, asymptotically tight (see Theorem 1.5 below), and has an elementary proof on the level of a graduate exercise. Yet, to our astonishment, it seems to be new. 
The inequality (7.1) should be compared to

$$
c_{\mu, \nu}^{\lambda} \leq f^{\nu} \quad \text { and } \quad c_{\mu, \nu}^{\lambda} \leq \frac{f^{\lambda / \mu}}{f^{\nu}}
$$

(see \$4.8), which are cleaner but asymptotically much weaker.

Note also that our proof of (7.1) can be phrased as a direct combinatorial argument, using a double counting surjection. Formally, the inequality

$$
f^{\lambda} f^{\mu} f^{\nu}\left(c_{\mu, \nu}^{\lambda}\right)^{2} \leq f^{\lambda} f^{\mu} f^{\nu}\left(\begin{array}{l}
n \\
k
\end{array}\right)
$$

is a combination of two surjections, both of which can be obtained by a jeu-de-taquin argument using two different combinatorial interpretation of $c_{\mu, \nu}^{\lambda}$ in terms of Young tableaux (cf. [Ker, vL, Zel]). We leave the details to the reader.

7.5. For small values of $n$ and $k$, the numerical values of the lower and upper bounds given by Proposition 4.4 are too far apart to be useful. For example, $\mathbf{C}(20,7)=11$ (see $₫$ A), while the proposition gives

$$
0.28 \approx \frac{1}{\sqrt{p(7) p(13) p(20)}} \sqrt{\left(\begin{array}{c}
20 \\
7
\end{array}\right)} \leq \mathbf{C}(20,7) \leq \sqrt{\left(\begin{array}{c}
20 \\
7
\end{array}\right)} \approx 278.42 .
$$

Even the upper bound $\mathbf{C}(20,7) \leq \mathbf{D}(7)=35 \leq \sqrt{7 !} \approx 70.99$ in Theorem 4.22 is better in this case.

7.6. By analogy with the upper bound in (2.4), we believe that $\mathbf{C}(n)$ are rather small when compared to $2^{n / 2}$. We conjecture that

$$
\mathbf{C}(n) \leq 2^{n / 2} e^{-a \sqrt{n}} \text { for some } a>0 .
$$

This suggests that $\mathbf{C}(n)$ is somewhere in between the lower and upper bound in Proposition 4.4. Note that Conjecture 5.5 comes close: it implies the upper bound $2^{n / 2} e^{-a \sqrt{n} / \log n}$, which would also be interesting.

7.7. We conjecture that Theorem 1.7 can be extended to general $0<\theta<1$. For that, one can try to improve the lower bound in Theorem 5.3 (see also Remark 5.4). This problem has an especially elegant special case in terms of Biane's approach [Bia], which can be presented as follows.

Let $\left\{\mu^{(n)} \vdash n / 2\right\}$ and $\left\{\nu^{(n)} \vdash n / 2\right\}$ be partition sequences with strongly stable shapes $\omega$ and $\pi$, respectively. Suppose area $(\omega)=\operatorname{area}(\pi)$. Let $\phi=\omega \boxplus \pi$ be the free convolution of the limit shape functions (see [Bia] for definitions), and let $\left\{\lambda^{(n)} \vdash n\right\}$ be partitions sequence with strongly stable shape $\phi$. It is easy to see that Stanley's upper bound (**) in Theorem 1.1 in terms of hook integrals (see $\$ 2.4$ ), combined with Biane's concentration results gives

$$
\Upsilon(\phi) \geq \frac{\Upsilon(\omega)+\Upsilon(\pi)}{2},
$$

with the equality for VKLS shapes.

Conjecture 7.1. Equality in (7.2) holds only for VKLS shapes. 
7.8. It would be interesting to sharpen our bounds in Theorem 4.13 for LR-coefficients with few rows. Perhaps, one can then ask for about the shape of the largest such LR-coefficients. Although our proof is combinatorial, our bounds are too far apart to be useful. Note also that this is analogous to the 1-parametric family of limit shapes with largest dimension and bound on the number of rows obtained by Logan and Shepp [LoS].

7.9. As we mentioned earlier there are no explicitly constructed series of partitions for which Kronecker coefficients are $\exp \Omega(n)$. In view of Theorem 1.4, it would be especially interesting to obtain an explicit construction giving a $\exp \Omega(n \log n)$. We conjecture that the staircase shapes in the Saxl conjecture give such examples, but as we mentioned in $\$ 3.5$, we are nowhere close to resolving this problem.

Acknowledgements. We are grateful to Richard Stanley for introducing us to the area; his questions and results were the inspiration for this whole paper. We would like to thank Sami Assaf, Vadim Gorin, Alejandro Morales, Leonid Petrov, Dan Romik, Dima Schlyaktenko and Martin Tassy for helpful comments and insightful remarks. The first and second authors were partially supported by the NSF. 


\section{REFERENCES}

[AR] R. Adin and Y. Roichman, Standard Young tableaux, in Handbook of Enumerative Combinatorics (M. Bóna, editor), CRC Press, Boca Raton, 2015, 895-974.

[AD] D. Aldous and P. Diaconis, Longest increasing subsequences, Bull. AMS 36 (1999), 413-432.

[BDJ] J. Baik, P. Deift and K. Johansson, On the distribution of the length of the longest increasing subsequence of random permutations, J. AMS 12 (1999), 1119-1178.

[BBR] F. Bergeron, R. Biagioli and M. H. Rosas, Inequalities between Littlewood-Richardson coefficients, J. Combin. Theory, Ser. A 113 (2006), 567-590.

[BB] C. Bessenrodt and C. Behns, On the Durfee size of Kronecker products of characters of the symmetric group and its double covers, J. Algebra 280 (2004), 132-144.

[Bia] Ph. Biane, Representations of Symmetric Groups and Free Probability, Adv. Math. 138 (1998), 126-181.

[BOR] E. Briand, R. Orellana and M. Rosas, The stability of the Kronecker product of Schur functions, J. Algebra 331 (2011), 11-27.

[Buf] A. I. Bufetov, On the Vershik-Kerov conjecture concerning the Shannon-McMillan-Breiman theorem for the Plancherel family of measures on the space of Young diagrams, Geom. Funct. Anal. 22 (2012), 938-975.

[BG] A. Bufetov and V. Gorin, Representations of classical Lie groups and quantized free convolution, Geom. Funct. Anal. 25 (2015), 763-814.

[Bür] P. Bürgisser, Permanent versus determinant, obstructions, and Kronecker coefficients, Sém. Lothar. Combin. 75 (2015-2017), Art. B75a, 19 pp.

[BI] P. Bürgisser and C. Ikenmeyer, Deciding positivity of Littlewood-Richardson coefficients, SIAM J. Discrete Math. 27 (2013), 1639-1681.

[CL] A. Chenciner and B. Leclerc, Between two moments, Regul. Chaotic Dyn. 19 (2014), 289-295.

[CDW] C. Chindris, H. Derksen and J. Weyman, Counterexamples to Okounkov's log-concavity conjecture, Compos. Math. 143 (2007), 1545-1557.

[DP] S. DeSalvo and I. Pak, Log-concavity of the partition function, Ramanujan J. 38 (2015), 61-73.

[Dvir] Y. Dvir, On the Kronecker product of $S_{n}$ characters, J. Algebra 154 (1993), 125-140.

[Ful] W. Fulton, Eigenvalues, invariant factors, highest weights, and Schubert calculus, Bull. AMS 37 (2000), 209-249.

[HW] P. E. Harris and J. F. Willenbring, Sums of squares of Littlewood-Richardson coefficients and GL $n^{-}$ harmonic polynomials, in Symmetry: representation theory and its applications, Birkhäuser, New York, 2014, 305-326.

[HSTZ] G. Heide, J. Saxl, P. H. Tiep and A. E. Zalesski, Conjugacy action, induced representations and the Steinberg square for simple groups of Lie type, Proc. LMS 106 (2013) 908-930.

[Ker] S. Kerov, The Robinson-Schensted-Knuth correspondence and the Littlewood-Richardson rule, Russian Math. Surv. 39 (1984) 165-166.

[KP] S. Kerov and A. M. Pass, Representations of symmetric groups with maximal dimension, J. Soviet Math. 59 (1992), 1131-1135.

[KT1] A. Knutson and T. Tao, The honeycomb model of $\mathrm{GL}_{n}(\mathbb{C})$ tensor products I: Proof of the saturation conjecture, J. AMS 12 (1999), 1055-1090.

[KT2] A. Knutson and T. Tao, Puzzles and (equivariant) cohomology of Grassmannians, Duke Math J. 119 (2003), 221-260.

[LPP $]$ T. Lam, A. Postnikov and P. Pylyavskyy, Schur positivity and Schur log-concavity, Amer. J. Math. 129 (2007), 1611-1622.

[LoS] B. F. Logan and L. A. Shepp, A variational problem for random Young tableaux, Adv. Math. 26 (1977), 206-222.

[LuS] S. Luo and M. Sellke, The Saxl conjecture for fourth powers via the semigroup property, J. Algebraic Combin. 45 (2017), 33-80.

[Man] L. Manivel, On the asymptotics of Kronecker coefficients, J. Algebraic Combin. 42 (2015), 999-1025.

[MPP1] A. H. Morales, I. Pak and G. Panova, Asymptotics of the number of standard Young tableaux of skew shape, Europ. J. Combin. 70 (2018), 26-49.

[MPP2] A. H. Morales, I. Pak and G. Panova, Hook formulas for skew shapes III. Multivariate and product formulas; arXiv:1707.00931. 
[MPT] A. H. Morales, I. Pak and M. Tassy, Asymptotics for the number of standard tableaux of skew shape and for weighted lozenge tilings; preprint (2018).

[Nar] H. Narayanan, Estimating deep Littlewood-Richardson coefficients, in Proc. FPSAC 2014, DMTCS, Nancy, France, 2014, 321-332

[PP1] I. Pak and G. Panova, Unimodality via Kronecker products, J. Algebraic Combin. 40 (2014), 11031120.

[PP2] I. Pak and G. Panova, Bounds on certain classes of Kronecker and $q$-binomial coefficients, J. Combin. Theory, Ser. A 147 (2017), 1-17.

[PPV] I. Pak, G. Panova and E. Vallejo, Kronecker products, characters, partitions, and the tensor square conjectures, Adv. Math. 288 (2016), 702-731.

[PPY] I. Pak, G. Panova and D. Yeliussizov, Bounds on the largest Kronecker and induced multiplicities of finite groups, preprint (2018).

[PV] I. Pak and E. Vallejo, Combinatorics and geometry of Littlewood-Richardson cones, emphEurop. J. Combin. 26 (2005), 995-1008.

[Reg] A. Regev, The Kronecker product of $S_{n}$-characters and an $A \otimes B$ theorem for Capelli identities, J. Algebra 66 (1980), 505-510.

[Rom] D. Romik, The surprising mathematics of longest increasing subsequences, Cambridge Univ. Press, New York, 2015.

[Sag] B. E. Sagan, The Symmetric Group, Springer, New York, 2001.

[SS1] B. E. Sagan and R. P. Stanley, Robinson-Schensted algorithms for skew tableaux, J. Combin. Theory, Ser. A 55 (1990), 161-193.

[SS2] S. V. Sam and A. Snowden, Proof of Stembridge's conjecture on stability of Kronecker coefficients, J. Algebraic Combin. 43 (2016), 1-10.

[OEIS] N. J. A. Sloane, The Online Encyclopedia of Integer Sequences, oeis.org

[S1] R. P. Stanley, Differential posets, J. AMS 1 (1988), 919-961.

[S2] R. P. Stanley, On the enumeration of skew Young tableaux, Adv. Appl. Math. 30 (2003), $283-294$.

[S3] R. P. Stanley, Increasing and decreasing subsequences and their variants, in Proc. ICM, Vol. I, EMS, Zürich, 2007, 545-579.

[S4] R. P. Stanley, Enumerative Combinatorics, vol. 1 (second ed.) and vol. 2, Cambridge Univ. Press, 2012 and 1999.

[S5] R. P. Stanley, Supplementary Excercies to [S4, available at http://www-math.mit.edu/ ${ }^{\sim} \mathrm{rstan} / \mathrm{ec}$

[S6] R. P. Stanley, Plethysm and Kronecker Products, talk slides, Sep. 26, 2016; available at http://www-math.mit.edu/ rstan/plethysm.pdf.

[vL] M. A. A. van Leeuwen, The Littlewood-Richardson rule, and related combinatorics, in Interaction of combinatorics and representation theory, Math. Soc. Japan, Tokyo, 2001, 95-145.

[Ver] A. M. Vershik, A statistical sum associated with Young diagrams, J. Soviet Math. 47 (1989), 23792386.

[VK1] A. M. Vershik and S. V. Kerov, The asymptotic character theory of the symmetric group, Funct. Anal. Appl. 15 (1981), 246-255.

[VK2] A. M. Vershik and S. V. Kerov, Asymptotic of the largest and the typical dimensions of irreducible representations of a symmetric group, Funct. Anal. Appl. 19 (1985), 21-31.

[VP] A. M. Vershik and D. Pavlov, Numerical experiments in problems of asymptotic representation theory, J. Math. Sci. 168 (2010), 351-361.

[Zel] A. V. Zelevinsky, A generalization of the Littlewood-Richardson rule and the Robinson-SchenstedKnuth correspondence, J. Algebra 69 (1981), 82-94. 
Here is the sequence $\{\mathbf{C}(n)\}$.

\begin{tabular}{|c|ccccccccccccccccccccccc|}
\hline$n$ & 1 & 2 & 3 & 4 & 5 & 6 & 7 & 8 & 9 & 10 & 11 & 12 & 13 & 14 & 15 & 16 & 17 & 18 & 19 & 20 & 21 & 22 & 23 \\
\hline $\mathbf{C}(n)$ & 1 & 1 & 1 & 1 & 1 & 2 & 2 & 2 & 2 & 3 & 3 & 4 & 4 & 5 & 6 & 8 & 9 & 11 & 12 & 18 & 24 & 32 & 35 \\
\hline
\end{tabular}

Here is the table of $\mathbf{C}(n, k)$, for $0 \leq k \leq 20$ and $1 \leq n \leq 23$. Note that $\mathbf{C}(n, k)=\mathbf{C}(n, n-k)$.

\begin{tabular}{|c|rrrrrrrrrrrrrrrrrrrrr|}
\hline$n \backslash k$ & 0 & 1 & 2 & 3 & 4 & 5 & 6 & 7 & 8 & 9 & 10 & 11 & 12 & 13 & 14 & 15 & 16 & 17 & 18 & 19 & 20 \\
\hline 1 & 1 & 1 & & & & & & & & & & & & & & & & & & \\
2 & 1 & 1 & 1 & & & & & & & & & & & & & & & & \\
3 & 1 & 1 & 1 & 1 & & & & & & & & & & & & & & & \\
4 & 1 & 1 & 1 & 1 & 1 & & & & & & & & & & & & & & \\
5 & 1 & 1 & 1 & 1 & 1 & 1 & & & & & & & & & & & & & \\
6 & 1 & 1 & 1 & 2 & 1 & 1 & 1 & & & & & & & & & & & & & \\
7 & 1 & 1 & 1 & 2 & 2 & 1 & 1 & 1 & & & & & & & & & & & & \\
8 & 1 & 1 & 1 & 2 & 2 & 2 & 1 & 1 & 1 & & & & & & & & & & & \\
9 & 1 & 1 & 1 & 2 & 2 & 2 & 2 & 1 & 1 & 1 & & & & & & & & & & \\
10 & 1 & 1 & 1 & 2 & 3 & 2 & 3 & 3 & 1 & 1 & 1 & & & & & & & & & \\
11 & 1 & 1 & 1 & 2 & 3 & 3 & 3 & 3 & 2 & 1 & 1 & 1 & & & & & & & & \\
12 & 1 & 1 & 1 & 2 & 3 & 3 & 4 & 3 & 3 & 2 & 1 & 1 & 1 & & & & & & & \\
13 & 1 & 1 & 1 & 2 & 3 & 3 & 4 & 4 & 3 & 3 & 2 & 1 & 1 & 1 & & & & & & \\
14 & 1 & 1 & 1 & 2 & 3 & 3 & 4 & 5 & 4 & 3 & 3 & 3 & 1 & 1 & 1 & & & & & \\
15 & 1 & 1 & 1 & 2 & 3 & 6 & 6 & 5 & 5 & 6 & 6 & 3 & 2 & 1 & 1 & 1 & & & & \\
16 & 1 & 1 & 1 & 2 & 3 & 6 & 8 & 7 & 6 & 7 & 8 & 6 & 3 & 2 & 1 & 1 & 1 & & & \\
17 & 1 & 1 & 1 & 2 & 3 & 6 & 8 & 9 & 8 & 8 & 9 & 8 & 6 & 3 & 2 & 1 & 1 & 1 & & \\
18 & 1 & 1 & 1 & 2 & 3 & 6 & 8 & 11 & 10 & 9 & 10 & 11 & 8 & 6 & 3 & 2 & 1 & 1 & 1 & & \\
19 & 1 & 1 & 1 & 2 & 3 & 6 & 8 & 11 & 12 & 11 & 11 & 12 & 11 & 8 & 6 & 3 & 2 & 1 & 1 & 1 & \\
20 & 1 & 1 & 1 & 2 & 3 & 6 & 8 & 11 & 12 & 13 & 18 & 13 & 12 & 11 & 8 & 6 & 3 & 2 & 1 & 1 & 1 \\
21 & 1 & 1 & 1 & 2 & 3 & 6 & 16 & 12 & 14 & 14 & 24 & 24 & 14 & 14 & 12 & 16 & 6 & 3 & 2 & 1 & 1 \\
22 & 1 & 1 & 1 & 2 & 3 & 6 & 16 & 20 & 15 & 16 & 27 & 32 & 27 & 16 & 15 & 20 & 16 & 6 & 3 & 2 & 1 \\
23 & 1 & 1 & 1 & 2 & 3 & 6 & 16 & 20 & 24 & 19 & 30 & 35 & 35 & 30 & 19 & 24 & 20 & 16 & 6 & 3 & 2 \\
\hline
\end{tabular}

For comparison, here is the sequence $\{\mathbf{D}(n)\}$ taken from OEIS, A003040, to which the column values in the table above stabilize by Theorem 4.22 .

\begin{tabular}{|c|cccccccccccccccc|}
\hline$n$ & 1 & 2 & 3 & 4 & 5 & 6 & 7 & 8 & 9 & 10 & 11 & 12 & 13 & 14 & 15 & 16 \\
\hline $\mathbf{D}(n)$ & 1 & 1 & 2 & 3 & 6 & 16 & 35 & 90 & 216 & 768 & 2310 & 7700 & 21450 & 69498 & 292864 & 1153152 \\
\hline
\end{tabular}

